\title{
The Potential Protective Effect of L-Ascorbic Acid against Chlorambucil Induced Hepatorenal Toxicity in Adults Male Albino Rats
}

\author{
Rabab Nabil Hafiz, Marwa M Fawzi ${ }^{1}$ and Ghada Galal Hamam².
}

${ }^{\mathbf{1}}$ Department of Forensic Medicine and Clinical Toxicology, Faculty of Medicine, Ain Shams University, Egypt.

${ }^{2}$ Department of Histology, Faculty of Medicine, Ain Shams University, Egypt

\begin{abstract}
:
The high incidence of neoplastic diseases have led to manifacturing of many antineoplastic drugs to combat these diseases. Treatment with chloambucil in high dose is associated with toxicities to many organs. The aim of this study is to investigate the protective role of L-ascorbic acid for prevention of chlorambucil induced hepatic and renal toxicities in rats. Methods: This study was conducted on 160 adult male albino rats divided into 8 equal groups . Group: I rats surved as negative control. Group II received L-ascorbic acid in an oral dose of $100 \mathrm{mg} / \mathrm{kg} /$ day . Group III received chlorambucil in an oral dose of $0.2 \mathrm{mg} / \mathrm{kg} /$ day for 5 consecutive days .Group IV received chlorambucil in the same dose for 10 days. Group V received chlorambucil in the same dose for 15 days. Group VI recieved chlorambucil and L-ascorbic acid in oral doses of $100 \mathrm{mg} / \mathrm{kg} / \mathrm{day}, 0.2 \mathrm{mg} / \mathrm{kg} /$ day respectively for 5 days. Group VII recieved chlorambucil and L-ascorbic acid in the same doses for 10 days. Group VIII recieved chlorambucil and L-ascorbic acid in the same doses for 15 days Results: There was significant difference in mean values of serum ALT ,AST, bilirubin, createnine, blood urea nitrogen and hepatic and renal GSH in groups III,IV and V compared to group I .Also, groups VI,VII and VIII showed significant difference in all studied parameters compared to group I and when groups VI,VII and VIII were compared with groups III,IV and V respectively, significant difference was also found. Histopathological examination of liver revealed severe damage in the form of congested dilated central veins and portal tracts ,vaculated hepatocytes and pyknotic nuclei in group III, these changes were more obvious in groups IV and V, the later revealed also mononuclear cellular infiltration. While there was mild damage in the form of mild congestion of the portal vein in group VI, dilated non congested portal veins in group VII and congestion of some central veins and some portal tracts with some hepatocytes with pyknotic nuclei in group VIII. Histopathological examination of kidneys revealed severe damage in the form of vacuolations of some renal tubules with some pyknotic nuclei in group III, loss of normal architecture of renal tubules in group IV and congested peritubular capillaries and frequently dilatated tubular lumen in group V. On the other hand, there was mild damage in the form of few tubular cells with pyknotic nuclei in group VI, vacuolations of some renal tubular cells in group VII and only congestion of peritubular capillaries in group VIII. while the percentage of pathological changes in liver and kidneys were insignificantly decreased in groups VI ,VII and VIII when compared with group I. Conclusion: Chlorambucil produced significant hepatorenal toxicities in time dependent manner. Co administration of Lascorbic acid improved such toxicities. This study was compared with other studies with larger number of experimental animals, using other antioxidants and affecting other organs. Recommendations: It is recommended to use L-ascorbic acid during therapy with chlorambucil in 5 days regimen and further studies about combined use of many antioxidants especially during prolonged therapy with chlorambucil are required.
\end{abstract}

Key words Acute toxicity, chlorambucil, L-ascorbic acid.

\section{Introduction}

Chlorambucil is an alkylating agent, it forms a covalent bond with proteins causing structural and functional damage to DNA especially of tumor cells so, it is used in therapy of oncologic diseases (Pangalis et al., 2002)

Chlorambucil (4-(4-(Bis(2-chlorethyl) amino) phenyl) butanoid acid) is the first choice to be used in treatment of chronic lymphocytic leukemia and low grade non Hodgkin's lymphoma ( $\mathrm{Li}$ et al., 2010) in a dose of $0.2 \mathrm{mg} / \mathrm{kg} / \mathrm{day}$ (high dose) for 5 consequative days in repeated cycles (ie 5 consequative days per week for 2 weeks with 2days in between, or for 3 weeks with 2 days in between, etc). (Balaram et al., 2012). It is used also in the same dose and manner as an immunosuppressant in nephrotic syndrome (Guigoniset 
al.,2008) and before bone marrow trasplantation in myeloma (Anagnostopoulos et al., 2006) and chronic After oral administration, chlorambucil is rapidly and totally absorbed from gastrointestinal tract, distributed to liver, kidneys and other organs.it is converted in liver by hepatic microsomal enzymes to 3(4-dehydro- Clorambucil) then to the final metabolite phenyl acetic acid mustard the later is further metabolized to inactive products excreted in urine and feces. (Pangalis et al., 2002)

The cytotoxic effects of chlorambucil are due to formation of ethylenimonium radical which causes damage of cellular antioxidant defense system in addition to breaks in DNA and cross linking of its double strands thus interfering with DNA replication and RNA transcription (Mc Evoyed,2006) .

The use of antioxidant in combination of chlorambucil may ameliorate its toxic effects (Singh et al.,2011)

\section{Aim of the study}

is to evaluate the potential protective role of L-ascorbic acid against chlorambucil induced hepatic and renal toxiciies in rats.

\section{Materials and methods}

The present study was conducted on 160 adult male albino rats weighing $200 \pm 50$ gm obtained from Egyptian Organization for Biological Products and Vaccines.They were kept in special animal cages under standardized conditions with free water supply and balanced diet and left for 14 days before the experiment for acclematization.

Ethical Consederations of The Study:

The experimental procedure was performed in accordance with the guide of the care and use of laboratory animal 's protocol approved by the Ethical Committee of Ain Shams University .All ethically approved conditions used by animal housing,feeding and handling were considered. The experimental protocol used followed the regulation for adminestration and painless sacrifaction for experimental animals.

\section{Drug, dose and route:}

Chlorambucil is a product of Aspen Pharma Trading Limited,Dublin ,Ireland, in the form of $2 \mathrm{mg}$ tablets each was grined and diluted in $10 \mathrm{ml}$ saline , $1 \mathrm{ml}$ of the solution was administrated to each rat orally by a gastric tube in a dose of $0.2 \mathrm{mg} / \mathrm{kg} /$ day for 5 consecutive days, and there was 2 days rest after each 5 consecutive days according to (Tomendedelova et al ., 2008).L-ascorbic acid was obtained from Unipharma in the form of $15 \mathrm{ml}$ solution in a dose of $100 \mathrm{mg} / \mathrm{kg} /$ day (non toxic dose) according to(Tunde et al., 2014) it was administrated also orally by a gastric tube.

\section{Animal grouping:}

The rats were classified into 8 equal groups,20 rats each.

Group I: (negative control group)

Group II: (L-ascorbic acid group): received L-Ascorbic acid in a dose of $100 \mathrm{mg} / \mathrm{kg} /$ day for 15 days.

Group III:received chlorambucil for 5 days in a dose of $0.2 \mathrm{mg} / \mathrm{kg} /$ day

Group IV: recieved chlorambucil for 10 days in a dose of $0.2 \mathrm{mg} / \mathrm{kg} / \mathrm{day}$ lymphocytic lymphoma with other chemotherapeutics (Tomendedelova et al., 2008).

Group V:received Chlorambucil for 15 days in a dose of $0.2 \mathrm{mg} / \mathrm{kg} /$ day

Group VI: received chlorambucil and L-Ascorbic acid for 5 days in doses of $0.2 \mathrm{mg} / \mathrm{kg} /$ day and $100 \mathrm{mg} / \mathrm{kg} /$ day respectively.

Group VII: received chlorambucil and L-Ascorbic acid for 10 days in doses of $0.2 \mathrm{mg} / \mathrm{kg} /$ day and $100 \mathrm{mg} / \mathrm{kg} /$ day respectively.

Group VIII: received chlorambucil and L-Ascorbic acid for 15 days in doses of $0.2 \mathrm{mg} / \mathrm{kg} / \mathrm{day}$ and $100 \mathrm{mg} / \mathrm{kg} /$ day respectively.

\section{Sample Collection:}

At the end of each experimental period, each rat group was anaesthesised by ether inhalation to avoid pain, dissection had been performed for all rats, $10 \mathrm{ml}$ blood samples were collected from abdominal aorta soon after death, in dry clean centrifuge tubes. Samples were centrifuged and serum was collected for assessing the biochemical parameters. kidneys and liver were dissected out and prepared for GSH assay and histopathological study by light microscopic examination.

Hepatic parameters: levels of Serum Aspartate Aminotransferase (ALT), Serum Alanine Aminotransferase (AST) were determined by colorimetric method according to Frankel and Gradwohl (1970) and total bilirubin was measured according to Suber (1994). The assay kits were purchased from Alkane Company.

Kidney parameters: Blood Urea Nitrogen (BUN) and serum createnine were measured by colorimetric method according to Lawrence and Robert (1993). The assay kits were purchased from Alkane Company.

\section{Histopathological examination:}

Glutathoine assay (GSH): Tissue samples were homogenized with ice-cold trichloroacetic acid (1gm tissue plus 10ml 10\%TCA)in an UltraTurrax tissue homogenizer. Glutathione measurements were performed using a modification of Ellman procedure (Ghosh et al; 2010). The assay kits were purchased from Alkane company.

The kidneys and the right lobe of the liver were rapidly removed from each animal. They were fixed in $10 \%$ formol-saline for one week. This was followed by dehydration, clearing and embedding in paraffin. Serial sections were cut at thickness of $5 \mu \mathrm{m}$ and were stained with hematoxylin and eosin stain (H\&E) (Bancroft et al., 1994) Sections were then examined and photographed in the Histology Department, Faculty of Medicine, Ain Shams University using Leica DM2500 microscope (Wetzlar, Germany) connected to a personal computer.

Statistical analysis:

The statistical analysis was performed using standard SPSS(st pacckage for social science)software package, version 20 (Chicago.IL)data were expressd as (mean \pm SD). Student's $t$-test ,ANOVA,one way statistical analysis and Chi square $\left(\mathrm{X}^{2}\right)$ were used to analyse the data with $\mathrm{p}<0.05$ and $\mathrm{q}>4.354$ considered statisticaly significant (Taylor,1990). 


\section{Results}

\section{Biochemical results}

The results depicted in tables 1,2,3,4,5 and 6 showed a comparison of studied biochemical parameters ; hepatic (AST,ALT and total serum bilirubin),renal(BUN and serum creatinine) and hepatic and renal GSH .Among all groups under study, there was insignificant difference in the mean values of all the tested parameters between ( group I ) control group and group II ( received L-ascorbic acid only).While, there was very high significant differense in the mean values of all studied parameters in chlorambucil groups as compared with the control group. Oral administration of L-ascorbic acid concomitantly with chlorambucil (group VI,VII and VIII)markedly increased hepatic and renal parameters and decreased hepatic and renal GSH. When compared with group I, regarding all studied parameters, group VI showed significant difference,group VII showed higher significant difference - except serum createnine where it showed very high significant difference-and group VIII showed very high significant difference.

When group III (received chlorambucil for 5 days) was compared with group VI(received L-ascorbic acid concomitantly with chlorambucil for 5 days), regarding hepatic biochemical parameters, high significant difference was observed.While regarding renal biochemical parameters and tissue GSH, a very high significant difference was found.

Comparing group IV(received chlorambucil for 10 days) and group VII (received L-ascorbic acid concomitantly with chlorambucil for 10 days), regarding all studied parameters, a high significant difference was observed (except serum creatinine and renal GSH where there was significant difference).On the other hand,a higher significant difference was observed regarding hepatic GSH.

Finally, group V(received chlorambucil for 15 days) was compared with group VIII(received Lascorbic acid concomitantly with chlorambucil for 15 days) and showed very high significant difference in all studied parameters except AST and BUN where high significant difference was observed .

\section{Histopathological results}

Examination of haematoxylin and eosin (H\&E) stained sections of the liver of control (group1) showed central vein in the middle of hepatic lobules and portal tract areas were seen in the periphery. Hepatocytes were polygonal in shape with acidophilic cytoplasm and were seen with vesicular nuclei. Some hepatocytes were seen binucleated. In-between hepatic cords, blood sinusoids were noticed. The portal areas were observed containing a branch from the portal vein, a branch from the hepatic artery and a bile duct. (Figs. $1,2)$

In chlorambucil treated rats for 5 days (Group III), congested dilated central veins and portal tract were observed. Vacuolated hepatocytes were also detected. Most hepatocytes were noticed with dense pyknotic nuclei . (Figs. 3,4 ). In chlorambucil treated rats for 10 days (Group IV), hepatocytes with cytoplasmic vacuolations and congested central veins were frequently noticed. Hepatocytes with abnormal pyknotic nuclei were also detected. (Figs. 5,6 ) While in chlorambucil treated rats for 15 days (Group V), extensive vacuolated hepatocytes with pyknotic nuclei were seen in most hepatic lobules. Dilated congested central veins, hepatic sinusoids and portal tracts were also detected. Areas of mononuclear cellular infiltration were also noticed. (Figs. 7,8,9)

The percentage of the pathological changes observed in groups III,IV and V showed significat difference from group I.(Table 7)

In rats given L-ascorbic acid concomitantly with chlorambucil for 5 days (group VI) there was mild congestion of the portal vein .(Figures 10,11) While those given L-ascorbic acid concomitantly with chlorambucil for 10 days (group VII) there was dilated non congested portal veins.(Figs. 12,13)

In rat group given L-ascorbic acid concomitantly with chlorambucil for 15 days (group VIII) there was congestion of some central veins and some portal tracts. Few hepatocytes were noticed with pyknotic nuclei (Figs. 14,15).

The percentage of pathological changes observed in groups VI,VII and VIII showed insignificant difference from group I except group VIII regarding central and portal veins congestion and dilatation where there was significant difference.(Table 7)

Examination of $H \& E$ stained kidney sections of control rats (Group I ) showed renal corpuscles with glomerular tuft of capillaries. Renal corpuscles were seen surrounded by Bowman's capsule. Proximal convoluted tubules were noticed with narrow lumina. They appeared lined by pyramidal cells with acidophilic cytoplasm and basal rounded vesicular nuclei. The distal convoluted tubules were observed with wide lumina. They were lined with low cubical cells with pale acidophilic cytoplasm and rounded vesicular nuclei. (Fig 16)

In rat group treated with chlorambucil for 5 days (Group III), vacuolations of some renal tubules were noticed. Some tubular cells showed pyknotic nuclei (Fig. 17). While those treated for 10 days (Group IV ), vacoulations of renal tubular cells, pyknotic nuclei and loss of normal architecture of renal tubules could also be observed (Fig. 18) and chlorambucil treated rats for 15 days (Group V), congestion of peritubular capillaries, and loss of normal tubular architecture with dilatation of tubular lumen were frequently found(Figs. 19,20)

The percentage of the pathological changes observed in groups III,IV and V showed significat difference from group I.(Table 8)

In rats given L-ascorbic acid concomitantly with chlorambucil for 5 days (Group VI), few tubular cells were appeared with pyknotic nuclei (Fig. 21).

In rats given L-ascorbic acid concomitantly with chlorambucil for 10 days (Group VII), vacuolations of some renal tubular cells were observed (Fig. 22).

While in rats given L-ascorbic acid concomitantly with chlorambucil for 15 days (Group VIII), congestion of peritubular capillaries could be 
detected (Fig.23).

The percentage of pathological changes observed in groups VI,VII and VIII showed insignificant difference from group I . (Table 8)

Table 1 ANOVA, One Way Statistical Analysis of serum AST ,ALT, and total serm bilirubin in studied groups (20 rats/group)

\begin{tabular}{|c|c|c|c|c|c|c|c|c|}
\hline & Group I & Group II & Group III & Group IV & Group V & Group VI & Group VII & Group VIII \\
\hline & $\begin{array}{c}\text { Mean } \pm \\
\text { SD }\end{array}$ & Mean \pm SD & Mean \pm SD & Mean \pm SD & Mean \pm SD & Mean \pm SD & $\begin{array}{c}\text { Mean } \pm \\
\text { SD }\end{array}$ & $\begin{array}{c}\text { Mean } \pm \\
\text { SD }\end{array}$ \\
\hline $\begin{array}{c}\text { AST } \\
\text { (IU/L) }\end{array}$ & $23.7 \pm 1$ & $\begin{array}{c}24.1 \pm 2 \\
\mathbf{p}>\mathbf{0 . 0 5} \\
\mathbf{q}=\mathbf{1 . 1 3 1}\end{array}$ & $\begin{array}{c}52 \pm 2 \\
\mathbf{p}<\mathbf{0 . 0 0 1} \\
\mathbf{q}=\mathbf{8 0 . 0 4 4}\end{array}$ & $\begin{array}{c}62 \pm 2 \\
\mathbf{p}<\mathbf{0 . 0 0 1} \\
\mathbf{q}=\mathbf{1 0 8 . 3 3}\end{array}$ & $\begin{array}{c}69 \pm 1 \\
\mathbf{p}<\mathbf{0 . 0 0 1} \\
\mathbf{q}=\mathbf{1 2 8 . 1 3}\end{array}$ & $\begin{array}{c}25.4 \pm 1 \\
\mathbf{p}<\mathbf{0 . 0 5} \\
\mathbf{q}=\mathbf{4 . 8 0 8}\end{array}$ & $\begin{array}{c}25.8 \pm 1 \\
\mathbf{p}<\mathbf{0 . 0 1} \\
\mathbf{q}=5.940\end{array}$ & $\begin{array}{c}38.7 \pm 2 \\
\mathbf{p}<\mathbf{0 . 0 0 1} \\
\mathbf{q}=\mathbf{4 2 . 4 2 6}\end{array}$ \\
\hline $\begin{array}{c}\text { ALT } \\
\text { (IU/L) }\end{array}$ & $22.8 \pm 2$ & $\begin{array}{c}23.1 \pm 3 \\
\mathbf{p}>\mathbf{0 . 0 5} \\
\mathbf{q}=\mathbf{0 . 4 7 7 2}\end{array}$ & $\begin{array}{c}64 \pm 3 \\
\mathbf{p}<\mathbf{0 . 0 0 1} \\
\mathbf{q}=\mathbf{6 8 . 2 6 2}\end{array}$ & $\begin{array}{c}68 \pm 2 \\
\mathbf{p}<\mathbf{0 . 0 0 1} \\
\mathbf{q}=\mathbf{7 4 . 9 1 2}\end{array}$ & $\begin{array}{c}69+ - \pm 2 \\
\mathbf{p}<\mathbf{0 . 0 0 1} \\
\mathbf{q}=\mathbf{7 6 . 5 6 9}\end{array}$ & $\begin{array}{c}25.8 \pm 1.5 \\
\mathbf{p}<\mathbf{0 . 0 5} \\
\mathbf{q}=\mathbf{4 . 7 0 7}\end{array}$ & $\begin{array}{l}26.2 \pm 1 \\
\mathbf{p}<\mathbf{0 . 0 1} \\
\mathbf{q}=\mathbf{5 . 6 3 5}\end{array}$ & $\begin{array}{c}42.7 \pm 52 \\
\mathbf{p}<\mathbf{0 . 0 0 1} \\
\mathbf{q}=\mathbf{3 2 . 8 8 1}\end{array}$ \\
\hline $\begin{array}{c}\text { Total Bil } \\
\text { (mg/dl) }\end{array}$ & $0.38 \pm 0.2$ & $\begin{array}{c}0.41 \pm 0.2 \\
\mathbf{p}>\mathbf{0 . 0 5} \\
\mathbf{q}=\mathbf{0 . 4 4 7}\end{array}$ & $\begin{array}{c}2.7 \pm 0.5 \\
\mathbf{p}<\mathbf{0 . 0 0 1} \\
\mathbf{q}=\mathbf{3 4 . 5 8 5}\end{array}$ & $\begin{array}{c}2.9 \pm 0.2 \\
\mathbf{p}<\mathbf{0 . 0 0 1} \\
\mathbf{q}=\mathbf{3 7 . 5 6 6}\end{array}$ & $\begin{array}{c}3.1 \pm 0.3 \\
\mathbf{p}<\mathbf{0 . 0 0 1} \\
\mathbf{q}=\mathbf{4 0 . 5 4 7}\end{array}$ & $\begin{array}{c}0.72 \pm 0.3 \\
\mathbf{p}<\mathbf{0 . 0 5} \\
\mathbf{q}=\mathbf{5 . 0 6 8}\end{array}$ & $\begin{array}{c}0.75 \pm 0.4 \\
\mathbf{p}<\mathbf{0 . 0 1} \\
\mathbf{q}=5.516\end{array}$ & $\begin{array}{c}0.1 .2 \pm 0.12 \\
\mathbf{p}<\mathbf{0 . 0 0 1} \\
\mathbf{q}=\mathbf{1 2 . 2 2 4}\end{array}$ \\
\hline
\end{tabular}

$p>0.05$ : insignificant difference; $p<0.05$ :significant difference; $p<0.01$ :high significant difference; $p<0.001$ :very high significant difference. $q>4.354$ : significant difference with group I.. Group I: (negative control group) Group II: (Lascorbic acid group):.Group III: chlorambucil for 5 days. Group IV: chlorambucil for 10 days. Group V: Chlorambucil for 15.Group VI: chlorambucil and L-Ascorbic acid for 5 days. Group VII: chlorambucil and L-Ascorbic acid for 10 days. Group VIII: chlorambucil and L-Ascorbic acid for 15 days.

Table 2 ANOVA, One Way Statistical Analysis of serum BUN and serum createnine in studied groups.(20 rats/group)

\begin{tabular}{|c|c|c|c|c|c|c|c|c|}
\hline & Group I & Group II & Group III & Group IV & Group V & Group VI & Group VII & Group VIII \\
\hline & $\begin{array}{c}\text { Mean } \pm \\
\text { SD }\end{array}$ & Mean \pm SD & $\begin{array}{c}\text { Mean } \pm \\
\text { SD }\end{array}$ & $\begin{array}{c}\text { Mean } \pm \\
\text { SD }\end{array}$ & $\begin{array}{c}\text { Mean } \pm \\
\text { SD }\end{array}$ & Mean \pm SD & $\begin{array}{c}\text { Mean } \pm \\
\text { SD }\end{array}$ & $\begin{array}{c}\text { Mean } \pm \\
\text { SD }\end{array}$ \\
\hline BUN(mg/dl) & $12.3 \pm 3$ & $\begin{array}{c}13.2 \pm \quad 2 \\
\mathbf{p}>0.05 \\
\mathbf{q}=1.090\end{array}$ & $\begin{array}{c}59.3 \pm 4 \\
\mathbf{p}<0.001 \\
q=56.9\end{array}$ & $\begin{array}{c}63.6 \pm 3 \\
\mathbf{p}<0.001 \\
\mathbf{q}=62.15\end{array}$ & $\begin{array}{c}69.6 \pm 3 \\
\mathbf{p}<0.001 \\
\mathbf{q}=69.42\end{array}$ & $\begin{array}{l}16.2 \pm 1 \\
\mathbf{p}<0.05 \\
\mathbf{q}=4.72\end{array}$ & & $\begin{array}{c}19.2 \pm 5 \\
\mathbf{p}<0.001 \\
\mathbf{q}=\mathbf{8 . 1 3}\end{array}$ \\
\hline $\operatorname{Cr}(\mathrm{mg} / \mathrm{dl})$ & $0.33 \pm .02$ & $\begin{array}{c}0.46 \pm 0.1 \\
\mathbf{p}>0.05 \\
\mathbf{q}=2.301\end{array}$ & $\begin{array}{l}2.4 \pm 0.1 \\
\mathbf{p}<0.001 \\
q=36.63\end{array}$ & $\begin{array}{l}3.1 \pm 0.2 \\
\mathbf{p}<0.001 \\
\mathbf{q}=49.02\end{array}$ & $\begin{array}{l}3.6 \pm 0.03 \\
\mathbf{p}<0.001 \\
\mathbf{q}=57.86\end{array}$ & $\begin{array}{c}\mathbf{0 . 6 1} \pm 0.4 \\
\mathbf{p}<\mathbf{0 . 0 5} \\
\mathbf{q}=4.955\end{array}$ & $\begin{array}{l}0.69 \pm 0.3 \\
\mathbf{p}<0.001 \\
q=6.371\end{array}$ & $\begin{array}{c}0.97 \pm 0.4 \\
\mathbf{p}<0.001 \\
\mathbf{q}=11.326\end{array}$ \\
\hline
\end{tabular}

$p>0.05$ :insignificant difference; $p<0.05$ :significant difference; $p<0.01$ :high significant difference; $p<0.001$ :very high significant difference. $q>4.354$ : significant difference with group I.

Table 3 ANOVA, One Way Statistical Analysis of tissue GSH in studied groups.(20 rats/group)

\begin{tabular}{|c|c|c|c|c|c|c|c|c|}
\hline & Group I & Group II & Group III & Group IV & Group V & Group VI & Group VII & $\begin{array}{c}\text { Group } \\
\text { VIII }\end{array}$ \\
\hline & Mean \pm SD & Mean \pm SD & Mean \pm SD & Mean \pm SD & Mean \pm SD & Mean \pm SD & Mean \pm SD & Mean \pm SD \\
\hline $\begin{array}{c}\text { Liver } \\
\text { GSH(Mmol/g) }\end{array}$ & $2.1 \pm 0.1$ & $\begin{array}{l}2.3 \pm 0.1 \\
\mathbf{p}>0.05 \\
\mathbf{q}=2.92\end{array}$ & $\begin{array}{c}0.9 \pm 0.6 \\
\mathbf{p}<0.001 \\
\mathbf{q}=17.52\end{array}$ & $\begin{array}{c}\mathbf{0 . 7} \pm \mathbf{0 . 1} \\
\mathbf{p}<0.001 \\
\mathbf{q}=20.44\end{array}$ & $\begin{array}{c}\mathbf{0 . 5} \pm 0.1 \\
\mathbf{p}<0.001 \\
\mathbf{q}=23.36\end{array}$ & $\begin{array}{l}\mathbf{1 . 8} \pm \mathbf{0 . 1} \\
\mathbf{p}<\mathbf{0 . 0 5} \\
\mathbf{q}=\mathbf{4 . 3 8}\end{array}$ & $\begin{array}{l}1.7 \pm 0.5 \\
\mathbf{p}<0.01 \\
\mathbf{q}=5.84\end{array}$ & $\begin{array}{c}1.4 \pm 0.3 \\
\mathbf{p}<0.001 \\
\mathbf{q}=10.22\end{array}$ \\
\hline $\begin{array}{c}\text { Kidney GSH } \\
\text { (Mmol/g) }\end{array}$ & $1.5 \pm 0.1$ & $\begin{array}{l}1.4 \pm 0.1 \\
p>0.05 \\
q=1.49\end{array}$ & $\begin{array}{c}0.5 \pm 0.5 \\
\mathbf{p}<0.001 \\
q=14.9\end{array}$ & $\begin{array}{c}0.4 \pm 0.3 p<0.001 \\
q=16.39\end{array}$ & $\begin{array}{c}0.4 \pm 0.1 \\
p<0.001 \\
q=16\end{array}$ & $\begin{array}{l}1.2 \pm 0.1 \\
\mathbf{p}<0.05 \\
\mathbf{q}=4.47\end{array}$ & $\begin{array}{l}1.1 \pm 0.5 \\
p<0.01 \\
q=5.69\end{array}$ & $\begin{array}{c}1 \pm 0.3 \\
\mathbf{p}<0.001 \\
\mathbf{q}=7.45\end{array}$ \\
\hline
\end{tabular}

$p>0.05$ : insignificant difference; $p<0.05$ :significant difference; $p<0.01$ :high significant difference; $p<0.001$ :very high significant difference. $q>4.354$ : significant difference with group I .

Table 4 Student ' $t$ ' test, analysis of serum AST, ALT and bilirubin in studied groups (20 rats/group)

\begin{tabular}{|c|c|c|c|c|c|c|c|c|c|c|c|c|}
\hline & Group III & Group VI & $\mathbf{t}$ & $\mathbf{p}$ & Group IV & Group VII & $\mathbf{t}$ & $\mathbf{p}$ & Group V & Group VIII & t & p \\
\hline & Mean \pm SD & Mean \pm SD & & & Mean \pm SD & Mean \pm SD & & & Mean \pm SD & Mean \pm SD & & \\
\hline AST(IU/L) & $52 \pm 2$ & $25.4 \pm 1$ & 53 & $<0.01$ & $62 \pm 2$ & $25.8 \pm 1$ & 72 & $<0.01$ & $69 \pm 1$ & $38.7 \pm 2$ & 60 & $<0.01$ \\
\hline ALT(IU/L) & $64 \pm 3^{*}$ & $25.8 \pm 1.5$ & 50 & $<0.01$ & $68 \pm 2$ & $26.2 \pm 1$ & 83 & $<0.01$ & $69+ - \pm 2$ & $42.7 \pm 5$ & 21 & $<0.001$ \\
\hline $\begin{array}{c}\text { Total } \\
\text { Bil(mg/dl) }\end{array}$ & $2.7 \pm 0.5$ & $0.72 \pm 0.3$ & 15 & $<0.01$ & $2.9 \pm 0.2$ & $0.75 \pm 0.4$ & 21 & $<0.01$ & $3.1 \pm 0.3$ & $1.2 \pm 0.1$ & 26 & $<0.001$ \\
\hline
\end{tabular}

$t$ :student $t$ test $; p>0.05$ :insignificant difference; $p<0.05$ :significant difference; $p<0.01$ :high significant difference; $p<0.001$ :very high significant difference. 
Table 5 Student 't' test analysis of BUN, serum createnine in studied groups (20 rats/group)

\begin{tabular}{|c|c|c|c|c|c|c|c|c|c|c|c|c|}
\hline & Group III & Group VI & $\mathbf{t}$ & $\mathbf{P}$ & $\begin{array}{c}\text { Group } \\
\text { IV }\end{array}$ & $\begin{array}{c}\text { Group } \\
\text { VII }\end{array}$ & $\mathbf{t}$ & $\mathbf{p}$ & Group V & $\begin{array}{c}\text { Group } \\
\text { VIII }\end{array}$ & $\mathbf{t}$ & $\mathbf{p}$ \\
\hline & Mean \pm SD & Mean \pm SD & & & Mean \pm SD & Mean \pm SD & & & Mean \pm SD & Mean \pm SD & & \\
\hline $\begin{array}{c}\text { BUN } \\
\text { (mg/dl) }\end{array}$ & $59.3 \pm 4$ & $16.2 \pm 1$ & 46 & $<0.001$ & $63.6 \pm 3$ & $16.9 \pm 6$ & 31 & $<0.01$ & $69.6 \pm 3$ & $19.2 \pm 5$ & 38 & $<0.01$ \\
\hline $\begin{array}{c}\mathrm{Cr} \\
(\mathrm{mg} / \mathrm{dl})\end{array}$ & $2.4 \pm 0.1$ & $0.61 \pm 0.4$ & 29 & $<0.001$ & $3.1 \pm 0.2$ & $0.69 \pm 0.3$ & 19 & $<0.05$ & $3.6 \pm 0.03$ & $0.97 \pm 0.4$ & 29 & $<0.001$ \\
\hline
\end{tabular}

t:student $t$ test ; $>0.05$ :insignificant difference; $p<0.05$ :significant difference; $p<0.01$ :high significant difference; $p<0.001$ :very high significant difference.

Table 6 Student 't' test analysis of tissue GSH in studied groups (20 rats/group)

\begin{tabular}{|c|c|c|c|c|c|c|c|c|c|c|c|c|}
\hline & Group III & Group VI & $\mathbf{t}$ & $\mathbf{p}$ & $\begin{array}{c}\text { Group } \\
\text { IV }\end{array}$ & $\begin{array}{c}\text { Group } \\
\text { VII }\end{array}$ & $\mathbf{t}$ & $\mathbf{p}$ & Group V & $\begin{array}{c}\text { Group } \\
\text { VIII }\end{array}$ & $\mathbf{t}$ & $\mathbf{p}$ \\
\hline & Mean \pm SD & Mean \pm SD & & & Mean \pm SD & Mean \pm SD & & & Mean \pm SD & Mean \pm SD & & \\
\hline $\begin{array}{c}\text { Liver GSH } \\
\text { (Mmol/g) }\end{array}$ & $0.9 \pm 0.6$ & $1.8 \pm 0.1$ & 6 & $<0.001$ & $0.7 \pm 0.1$ & $1.7 \pm 0.5$ & 8 & $<0.001$ & $0.5 \pm 0.1$ & $1.4 \pm 0.3$ & 12 & $<0.001$ \\
\hline $\begin{array}{c}\text { Kidney } \\
\text { GSH } \\
\text { (Mmol/g) }\end{array}$ & $0.5 \pm 0.5$ & $1.2 \pm 0.1$ & 6 & $<0.001$ & $0.4 \pm 0.3$ & $1.1 \pm 0.5$ & 5 & $<0.05$ & $0.4 \pm 0.1$ & $1 \pm 0.3$ & 8 & $<0.001$ \\
\hline
\end{tabular}

t:student $t$ test $; p>0.05$ :insignificant difference; $p<0.05$ :significant difference; $p<0.01$ :high significant difference; $p<0.001$ :very high significant difference.

Table 7 Chi square test $\left(\mathrm{X}^{2}\right)$ analysis of hepatic histopathological findings in studied groups.(20 rats/group)

\begin{tabular}{|c|c|c|c|c|c|c|c|c|}
\hline & Group & Group II & Group III & Group IV & Group V & Group VI & Group VII & $\begin{array}{l}\text { Group } \\
\text { VIII }\end{array}$ \\
\hline & No. \% & No. $\%$ & No. $\%$ & No. $\%$ & No. $\%$ & No. $\%$ & No. $\%$ & No. $\%$ \\
\hline $\begin{array}{c}\text { Central vein } \\
\text { congestion } \\
\text { \&dilatation }\end{array}$ & 10 & $\begin{array}{cc}1 & 5 \\
\mathbf{X}^{2} & \mathbf{0 . 3 6} \\
\mathbf{P}>\mathbf{0 . 0 5} \\
\end{array}$ & $\begin{array}{c}1785 \\
X^{2} 22.55 \\
\mathbf{p}<\mathbf{0 . 0 5}\end{array}$ & $\begin{array}{cc}17 & 85 \\
\mathbf{X}^{2} & \mathbf{2 2 . 5 5} \\
\mathbf{P}<\mathbf{0 . 0 5} \\
\end{array}$ & $\begin{array}{cr}19 & 95 \\
\mathbf{X}^{2} & \mathbf{2 8 . 9} \\
\mathbf{p}<\mathbf{0 . 0 5}\end{array}$ & $\begin{array}{lr}5 & 25 \\
X^{2} & \mathbf{0 . 1 5} \\
\mathbf{p}>\mathbf{0 . 0 5}\end{array}$ & $\begin{array}{cc}6 & 30 \mathrm{X}^{2} \\
& \mathbf{1 . 6} \\
\mathbf{p}>\mathbf{0 . 0 5} \\
\end{array}$ & $\begin{array}{cc}7 & 35 \\
\mathbf{X}^{2} & \mathbf{0 . 3 5} \\
\mathbf{P}<\mathbf{0 . 0 5} \\
\end{array}$ \\
\hline $\begin{array}{l}\text { Portal tract } \\
\text { congestion } \\
\text { \&dilatation }\end{array}$ & 1 & $\begin{array}{c}3 \quad 15 \mathrm{X}^{2} \\
\mathbf{1 . 1} \\
\mathbf{p}>\mathbf{0 . 0 5}\end{array}$ & $\begin{array}{lr}14 & 70 \\
\mathbf{X}^{2} & \mathbf{2 4} \\
\mathbf{p}<\mathbf{0 . 0 5}\end{array}$ & 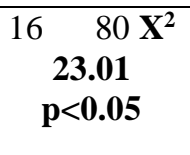 & $\begin{array}{cr}18 & 90 \\
\mathbf{X}^{2} & \mathbf{3 4 . 7} \\
\mathbf{p}<\mathbf{0 . 0 5}\end{array}$ & $\begin{array}{lr}4 & 20 \\
\mathbf{X}^{2} & \mathbf{0 . 2 1} \\
\mathbf{p}>\mathbf{0 . 0 5}\end{array}$ & $\begin{array}{cc}5 & 25 \mathrm{X}^{2} \\
& \mathbf{1 . 2} \\
\mathbf{p}> & \mathbf{0 . 0 5}\end{array}$ & $\begin{array}{l}5 \quad 25 \\
\mathbf{X}^{2} 2.7 \\
\mathbf{p}<\mathbf{0 . 0 5}\end{array}$ \\
\hline $\begin{array}{l}\text { Portal vein } \\
\text { congestion }\end{array}$ & 2 & $\begin{array}{cc}0 & 0 \\
\mathbf{X}^{2} & \mathbf{0} \\
\mathbf{p}>\mathbf{0 . 0 5}\end{array}$ & $\begin{array}{c}16 \quad 80 \mathbf{X}^{2} \\
\mathbf{1 7} \\
\mathbf{p}<\mathbf{0 . 0 5} \\
\end{array}$ & $\begin{array}{cr}17 & 85 \\
\mathbf{X}^{2} & \mathbf{2 2 . 5 5} \\
\mathbf{p} & <\mathbf{0 . 0 5}\end{array}$ & $\begin{array}{cr}19 & 95 \\
\mathbf{X}^{2} & \mathbf{2 8 . 9} \\
\mathbf{p}<\mathbf{0 . 0 5}\end{array}$ & $\begin{array}{cc}2 & 10 \\
\mathbf{X}^{2} & \mathbf{0} \\
\mathbf{p}>\mathbf{0 . 0 5}\end{array}$ & $\begin{array}{lr}4 & 20 \\
\mathbf{X}^{\mathbf{2}} \mathbf{0 . 0 7} \\
\mathbf{p}>\mathbf{0 . 0 5}\end{array}$ & $\begin{array}{lr}5 & 25 \\
\mathbf{X}^{2} & \mathbf{1 . 5} \\
\mathbf{p}>\mathbf{0 . 0 5}\end{array}$ \\
\hline $\begin{array}{l}\text { Portal vein } \\
\text { dilatatoin }\end{array}$ & 0 & $\begin{array}{cc}1 & 5 \\
\mathbf{X}^{2} & \mathbf{0} \\
\mathbf{p}>\mathbf{0 . 0 5}\end{array}$ & $\begin{array}{c}15 \quad 75 \mathbf{X}^{2} \\
\mathbf{2 4} \\
\mathbf{p}<\mathbf{0 . 0 5} \\
\end{array}$ & $\begin{array}{l}16 \quad 80 X^{2} \\
31.2 \mathbf{p}<\mathbf{0 . 0 5}\end{array}$ & $\begin{array}{cc}17 & 85 \\
\mathbf{X}^{2} & \mathbf{2 9 . 0 4} \\
\mathbf{p}<\mathbf{0 . 0 5}\end{array}$ & $\begin{array}{lr}3 & 15 \\
X^{2} & \mathbf{0 . 2 5} \\
\mathbf{p}>\mathbf{0 . 0 5}\end{array}$ & $\begin{array}{lr}6 & 30 \\
\mathbf{X}^{\mathbf{2}} & \mathbf{0 . 7} \\
\mathbf{p}>\mathbf{0 . 0 5}\end{array}$ & $\begin{array}{lr}8 & 40 \\
X^{2} & 0.73 \\
\mathbf{p}> & \mathbf{0 . 0 5}\end{array}$ \\
\hline $\begin{array}{l}\text { Hepatocytes } \\
\text { vaculation }\end{array}$ & 1 & $\begin{array}{ll}2 & 10 \\
\mathbf{X}^{2} & \mathbf{0} \\
\mathbf{p}>\mathbf{0 . 0 5}\end{array}$ & $\begin{array}{cc}13 & 65 \\
\mathbf{X}^{\mathbf{2}} & \mathbf{1 5 . 8} \\
\mathbf{p}<\mathbf{0 . 0 5}\end{array}$ & $\begin{array}{cc}15 & 75 \\
\mathbf{X}^{2} & \mathbf{2 0 . 4} \\
\mathbf{p}<\mathbf{0 . 0 5}\end{array}$ & $\begin{array}{cr}16 & 80 \\
X^{2} & \mathbf{2 4 . 4} \\
\mathbf{p}<\mathbf{0 . 0 5}\end{array}$ & $\begin{array}{lr}4 & 25 \\
X^{2} & 0.92 \\
\mathbf{p}>\mathbf{0 . 0 5}\end{array}$ & $\begin{array}{lc}4 \quad 25 \mathrm{X}^{\mathbf{2}} \\
\mathbf{0 . 4} \\
\mathbf{p}>\mathbf{0 . 0 5}\end{array}$ & $\begin{array}{lr}6 & 30 \\
\mathbf{X}^{2} & \mathbf{1 . 4} \\
\mathbf{p}>\mathbf{0 . 0 5}\end{array}$ \\
\hline $\begin{array}{l}\text { Hepatocytes } \\
\text { pyknosis }\end{array}$ & 0 & $\begin{array}{lr}1 & 5 X^{2} \\
0 & p>0.05\end{array}$ & $\begin{array}{lr}12 & 60 \\
\mathbf{X}^{2} & \mathbf{1 3 . 6} \\
\mathbf{p}<\mathbf{0 . 0 5}\end{array}$ & $\begin{array}{cc}14 & 70 \\
\mathbf{X}^{\mathbf{2}} & \mathbf{2 0 . 7} \\
\mathbf{p}<\mathbf{0 . 0 5}\end{array}$ & $\begin{array}{cr}15 & 75 \\
X^{2} & \mathbf{2 0 . 6} \\
\mathbf{p}<\mathbf{0 . 0 5}\end{array}$ & $\begin{array}{lr}6 & 30 \\
X^{2} & \mathbf{0 . 1} \\
\mathbf{p}>\mathbf{0 . 0 5}\end{array}$ & $\begin{array}{cc}6 & 30 \mathrm{X}^{2} \\
& \mathbf{0 . 6} \\
\mathbf{p}>\mathbf{0 . 0 5} \\
\end{array}$ & $\begin{array}{lr}7 & 35 \\
\mathbf{X}^{\mathbf{2}} & \mathbf{0 . 8} \\
\mathbf{p}>\mathbf{0 . 0 5}\end{array}$ \\
\hline $\begin{array}{l}\text { Mononuclear cell } \\
\text { infilteration }\end{array}$ & 3 & $\begin{array}{cc}1 & 5 \\
\mathbf{X}^{2} & \mathbf{0 . 3 4} \\
\mathbf{p}>\mathbf{0 . 0 5} \\
\end{array}$ & $\begin{array}{cr}14 & 70 \\
\mathbf{X}^{2} & \mathbf{1 8 . 0 2} \\
\mathbf{p}<\mathbf{0 . 0 5}\end{array}$ & $\begin{array}{cc}16 \quad 80 \mathrm{X}^{\mathbf{2}} \\
\mathbf{2 0 . 3} \\
\mathbf{p}<\mathbf{0 . 0 5}\end{array}$ & $\begin{array}{cc}18 & 90 \\
X^{2} & \mathbf{1 6 . 9 4} \\
\mathbf{p}<\mathbf{0 . 0 5}\end{array}$ & $\begin{array}{lr}4 & 25 \\
\mathbf{X}^{2} & \mathbf{0 . 2} \\
\mathbf{p}>\mathbf{0 . 0 5}\end{array}$ & $\begin{array}{lr}7 & 35 \\
\mathbf{X}^{\mathbf{2}} & \mathbf{0 . 8} \\
\mathbf{p}>\mathbf{0 . 0 5}\end{array}$ & $\begin{array}{cc}9 & 45 \\
X^{2} & \mathbf{0 . 1 4} \\
\mathbf{p}>\mathbf{0 . 0 5}\end{array}$ \\
\hline
\end{tabular}

Where $X^{2}$ :chi square. level of significance $\alpha-0.05$ where $p>0.05$ :insegnificant difference; $p<0.05$ :significant difference. 
Table 8 Chi square test $\left(\mathrm{X}^{2}\right)$ in studied groups.(20 rats/group)

\begin{tabular}{|c|c|c|c|c|c|c|c|c|}
\hline & Group I & Group II & Group III & Group IV & Group V & Group VI & Group VII & $\begin{array}{c}\text { Group } \\
\text { VIII }\end{array}$ \\
\hline & No. $\%$ & No. $\%$ & No. \% & No. \% & No. \% & No. \% & No. \% & No. \% \\
\hline $\begin{array}{c}\text { Tubular cells } \\
\text { vaculation }\end{array}$ & 5 & $\begin{array}{cc}0 & 0 \\
\mathbf{X}^{2} & \mathbf{3 6} \\
\mathbf{p}>\mathbf{0 . 0 5}\end{array}$ & $\begin{array}{c}1575 \mathrm{X}^{2} \\
\mathbf{2 0 . 4} \\
\mathbf{p}<\mathbf{0 . 0 5}\end{array}$ & $\begin{array}{lr}17 & 85 \\
X^{2} & \mathbf{2 5 . 8} \\
\mathbf{p}<\mathbf{0 . 0 5}\end{array}$ & $\begin{array}{c}1890 \mathrm{X}^{2} \\
\mathbf{5 . 4} \\
\mathbf{p}<\mathbf{0 . 0 5}\end{array}$ & $\begin{array}{cc}4 & 20 \\
\mathbf{X}^{2} & \mathbf{0 . 2} \\
\mathbf{p}>\mathbf{0 . 0 5}\end{array}$ & $\begin{array}{c}5 \quad 25 \\
\mathrm{X}^{2} 0.3 \mathrm{p}> \\
0.05\end{array}$ & $\begin{array}{cc}7 & 35 \\
\mathbf{X}^{\mathbf{2}} & \mathbf{0 . 3} \\
\mathbf{p}>\mathbf{0 . 0 5}\end{array}$ \\
\hline $\begin{array}{l}\text { Tubular cells } \\
\text { pyknosis }\end{array}$ & 10 & $\begin{array}{cc}1 & 5 \\
X^{2} & \mathbf{2 6} \\
\mathbf{p}>\mathbf{0 . 0 5}\end{array}$ & $\begin{array}{cc}16 & 80 \\
\mathbf{X}^{2} & \mathbf{1 9 . 7} \\
\mathbf{p}<\mathbf{0 . 0 5}\end{array}$ & $\begin{array}{c}1785 \mathbf{X}^{2} \\
5.7 \\
\mathbf{p}<\mathbf{0 . 0 5}\end{array}$ & $\begin{array}{lr}19 & 95 \\
\mathbf{X}^{2} & \mathbf{5 . 4} \\
\mathbf{p}<\mathbf{0 . 0 5}\end{array}$ & $\begin{array}{c}5 \quad 25 \\
\mathbf{X}^{2} \mathbf{0 . 1 5} \mathrm{p}^{>} \\
0.05\end{array}$ & $\begin{array}{cc}6 & 30 \\
\mathbf{X}^{2} & \mathbf{0 . 2 5} \\
\mathbf{p}>\mathbf{0 . 0 5}\end{array}$ & $\begin{array}{cr}7 & 35 \\
\mathbf{X}^{\mathbf{2}} & \mathbf{1 . 9} \\
\mathbf{p}>\mathbf{0 . 0 5}\end{array}$ \\
\hline $\begin{array}{c}\text { Tubular lumen } \\
\text { dilatation }\end{array}$ & 0 & $\begin{array}{lr}0 & 0 \mathbf{X}^{2} \\
0 & \mathbf{p}>\mathbf{0 . 0 5}\end{array}$ & $\begin{array}{c}1470 \mathrm{X}^{2} \\
\mathbf{2 0 . 5} \\
\mathbf{p}<\mathbf{0 . 0 5}\end{array}$ & $\begin{array}{c}16 \quad 80 \\
X^{2} \mathbf{2 6 . 0 1} \\
\mathbf{P}<\mathbf{0 . 0 5}\end{array}$ & $\begin{array}{l}17 \quad 85 \\
X^{2} \\
\mathbf{2 9 . 5} \\
\mathbf{P}<\mathbf{0 . 0 5}\end{array}$ & $\begin{array}{c}5 \quad 25 \\
X^{2} 0.57 p> \\
0.05\end{array}$ & $\begin{array}{cc}8 & 40 \\
\mathbf{X}^{2} & \mathbf{0 . 8 5} \\
\mathbf{p}>\mathbf{0 . 0 5}\end{array}$ & $\begin{array}{rl}9 & 45 \\
\mathbf{X}^{2} & \mathbf{1 . 0 2} \\
\mathbf{p}>\mathbf{0 . 0 5}\end{array}$ \\
\hline $\begin{array}{c}\text { Peritubular } \\
\text { capillaries } \\
\text { congestion }\end{array}$ & 5 & $\begin{array}{c}210 \mathrm{X}^{2} \\
\mathbf{8 . 9} \\
\mathbf{p}>\mathbf{0 . 0 5}\end{array}$ & $\begin{array}{cr}15 & 75 \\
\mathbf{X}^{2} & \mathbf{1 4 . 3} \\
\mathbf{p}<\mathbf{0 . 0 5}\end{array}$ & $\begin{array}{c}16 \quad 80 \\
X^{2} \mathbf{2 3 . 0 1} \\
\mathbf{p}<\mathbf{0 . 0 5}\end{array}$ & $\begin{array}{lr}18 & 90 \\
\mathbf{X}^{2} & \mathbf{5 . 4} \\
\mathbf{p}<\mathbf{0 . 0 5}\end{array}$ & $\begin{array}{cc}3 & 15 \\
\mathbf{X}^{\mathbf{2}} & \mathbf{1 . 4} \\
\mathbf{p}>\mathbf{0 . 0 5}\end{array}$ & $\begin{array}{cc}4 & 20 \\
\mathbf{X}^{2} & \mathbf{0 . 2 1} \\
\mathbf{p}>\mathbf{0 . 0 5}\end{array}$ & $\begin{array}{cc}7 & 35 \\
\mathbf{X}^{\mathbf{2}} & \mathbf{0 . 3} \\
\mathbf{p}>\mathbf{0 . 0 5}\end{array}$ \\
\hline $\begin{array}{c}\text { Loss of normal } \\
\text { tubular } \\
\text { architicture }\end{array}$ & 5 & $\begin{array}{cc}1 & 5 X^{2} \\
32.4 \\
p>0.05\end{array}$ & $\begin{array}{lr}14 & 70 \\
\mathbf{X}^{2} & \mathbf{1 8} \\
\mathbf{p}<\mathbf{0 . 0 5} & \end{array}$ & $\begin{array}{c}16 \quad 80 \\
X^{2} \mathbf{2 3 . 0 1} \\
\mathbf{p}<\mathbf{0 . 0 5}\end{array}$ & $\begin{array}{c}1995 \mathrm{X}^{\mathbf{2}} \\
\mathbf{3 2 . 4} \mathbf{p}<\mathbf{0 . 0 5}\end{array}$ & $\begin{array}{cc}3 & 15 \\
\mathbf{X}^{2} & \mathbf{1 . 4} \mathbf{p}> \\
0.05\end{array}$ & $\begin{array}{cc}4 & 20 \\
\mathbf{X}^{2} & \mathbf{0 . 2 1} \\
\mathbf{p}>\mathbf{0 . 0 5}\end{array}$ & $\begin{array}{cc}5 & 25 \\
X^{2} & 0.3 \text { p }> \\
0.05\end{array}$ \\
\hline
\end{tabular}

Where $X^{2}$ :chi square. level of significance $\alpha-0.05$ where $p>0.05$ :insegnificant difference; $p<0.05$ :significant difference.

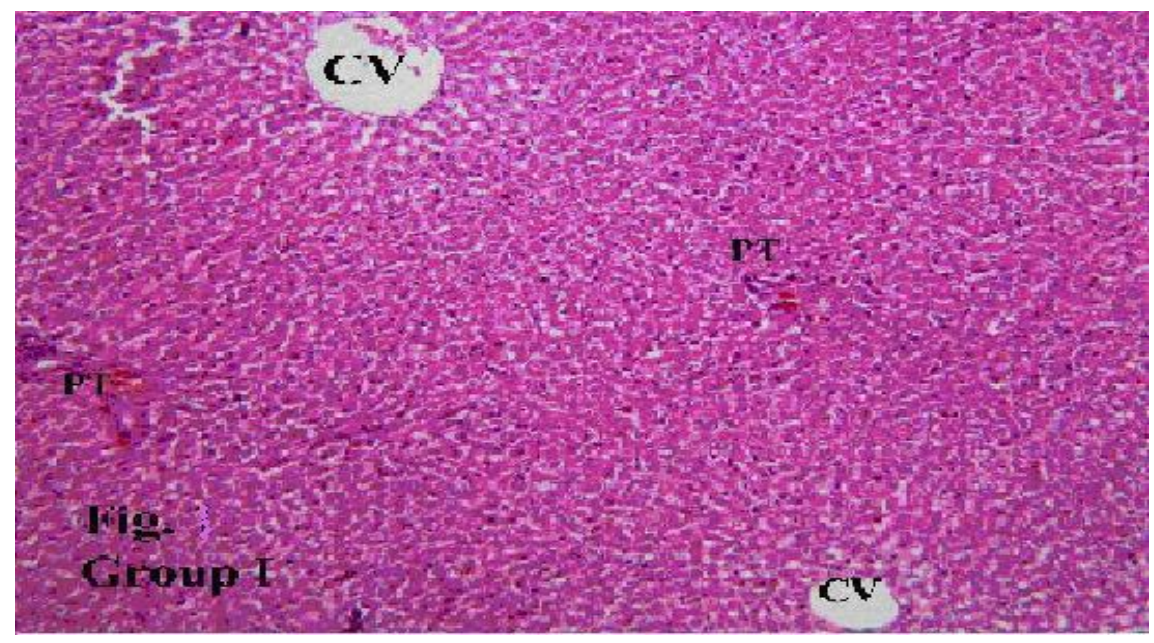

Figure 1: photomicrographs of rat liver sections (group I) showing: Central vein (CV) in the middle of hepatic lobules. Portal tract (PT) areas are seen in the periphery of hepatic lobules (H\&EX 250).

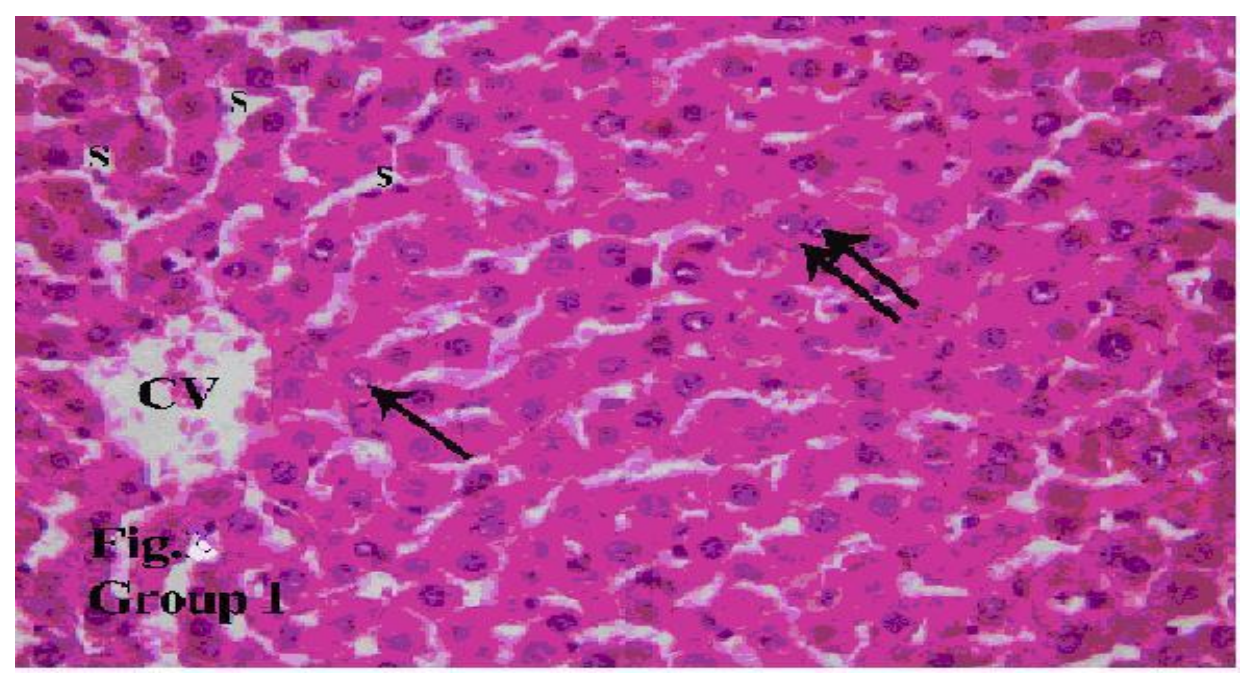

Figure 2: photomicrographs of rat liver sections (group I): showing: Central vein (CV), hepatocytes are seen with vesicular nuclei $(\uparrow)$. Binucleated cells $(\uparrow \uparrow)$ are also seen. In-between hepatic cords, blood sinusoids (S) are seen (H\&E X 640) 


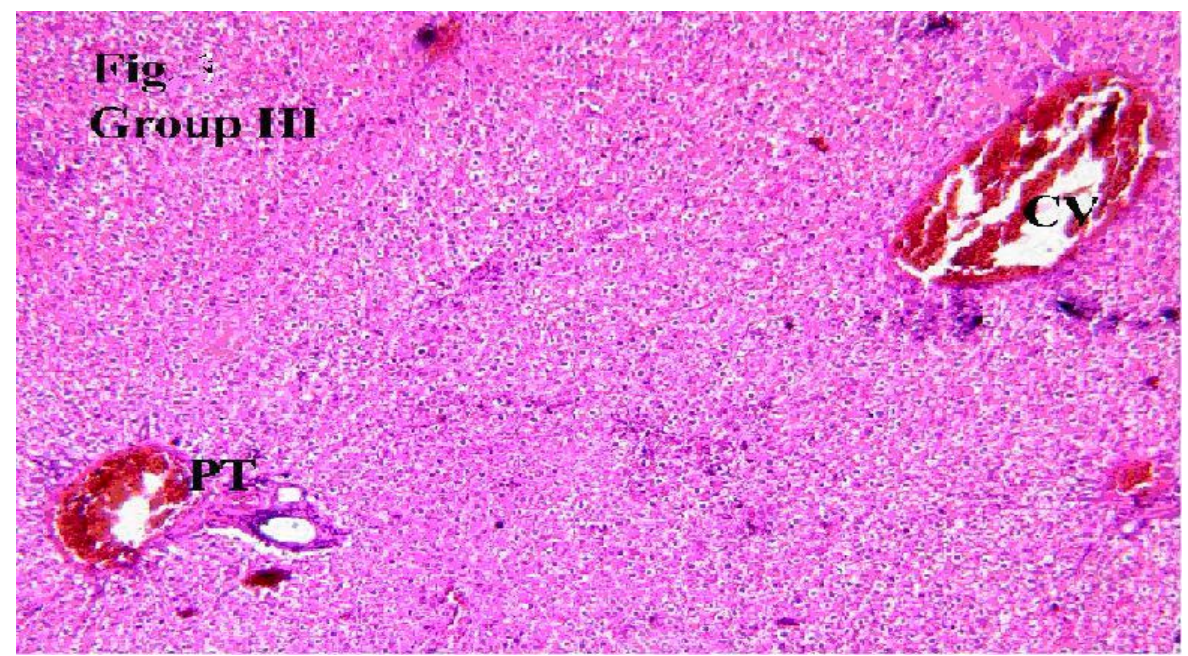

Figure 3: photomicrographs of rat liver sections (group III):, showing congested dilated central vein (CV) and portal tract (PT). (H\&E X 250).

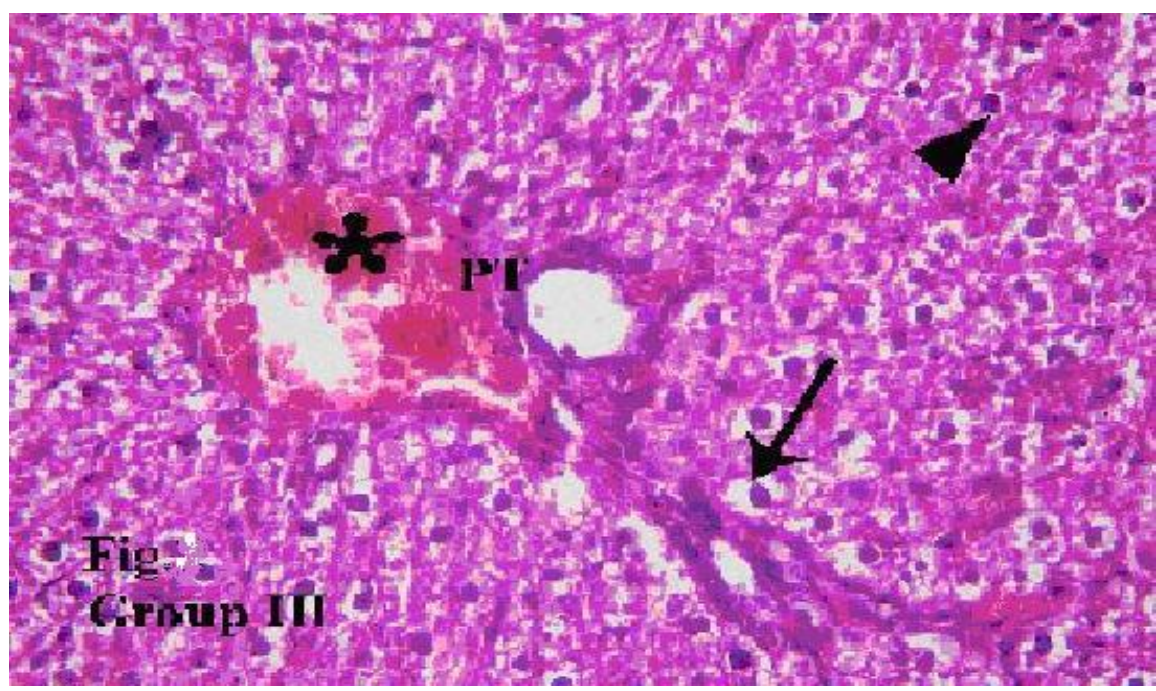

Figure 4 photomicrographs of rat liver sections (group III): showing vacuolated hepatocytes ( $\uparrow$ ) near a dilated portal tract (PT). Congested portal vein (*) can be seen. Most hepatocytes are seen with dense pyknotic nuclei ( $)$ ).(H\&E X 640).

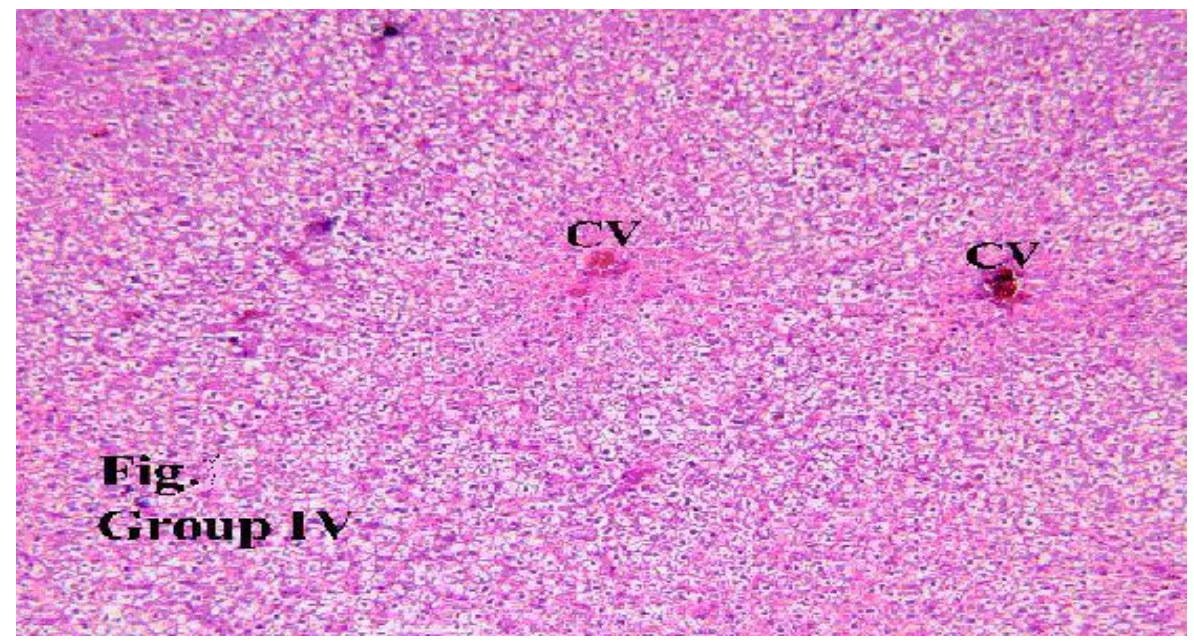

Figure 5: photomicrographs of rat liver sections (group IV): showing hepatocytes with cytoplasmic vacuolations. Congested central veins (CV) are also seen (H\&E X 250). 


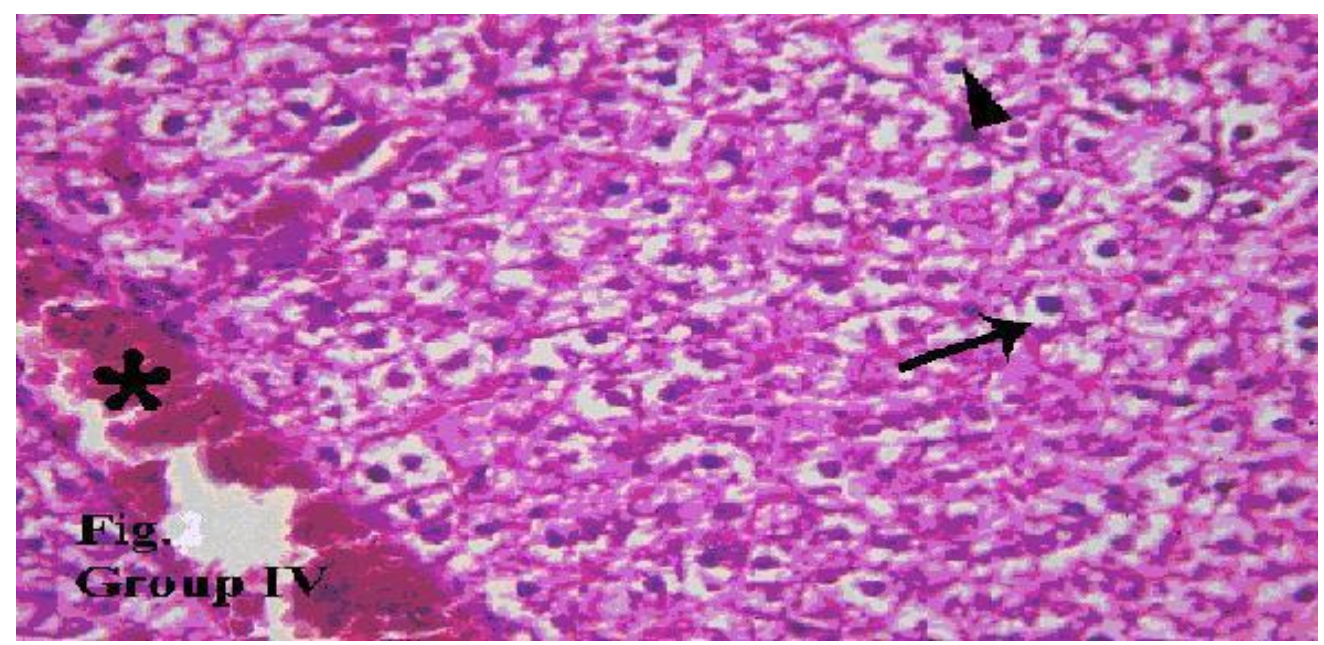

Figure 6 photomicrographs of rat liver sections (group IV): showing hepatocytes with extensive cytoplasmic vacuolations $(\uparrow)$. Hepatocytes with abnormal pyknotic nuclei $(\Delta)$ and dilated congested portal vein $(*)$ can also be seen. (H\&E X 640).

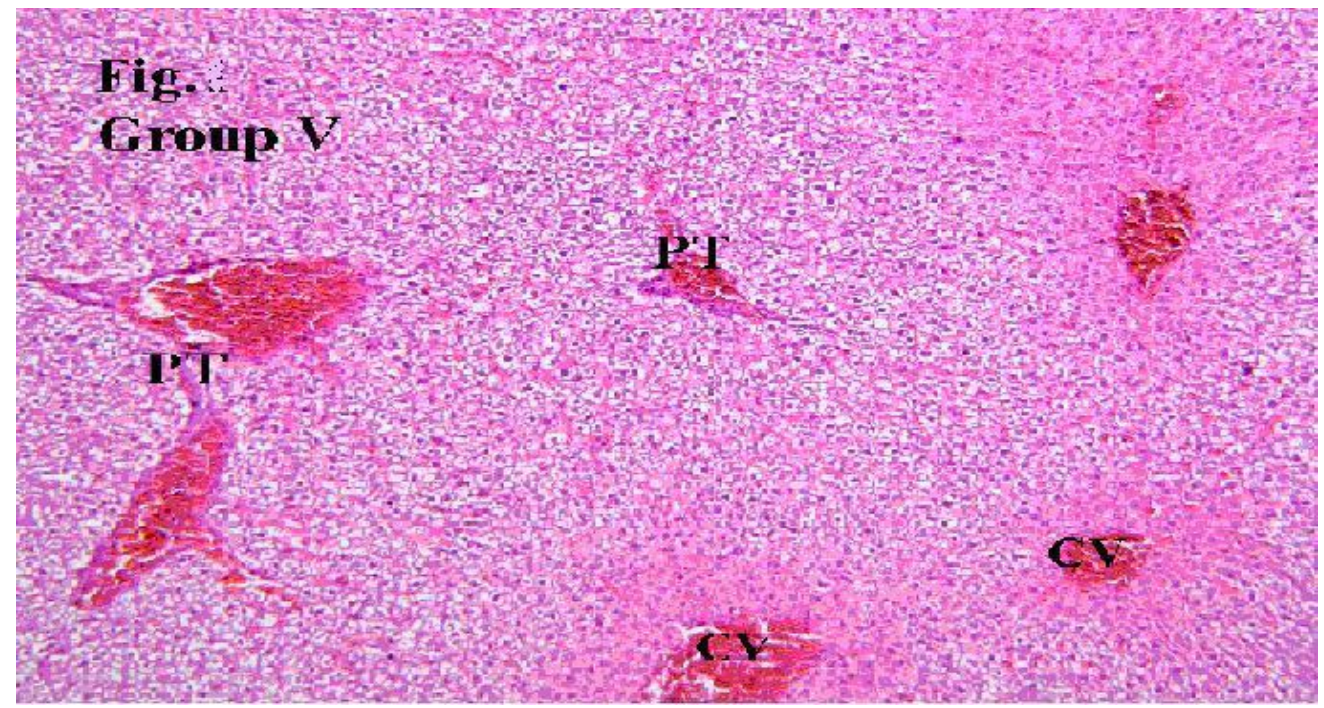

Figure 7: photomicrographs of rat liver sections (group V): showing extensive vacuolated hepatocytes. Dilated congested central vein $(C V)$ and portal tracts $(P T)$ are noticed $(H \& E X 250)$.

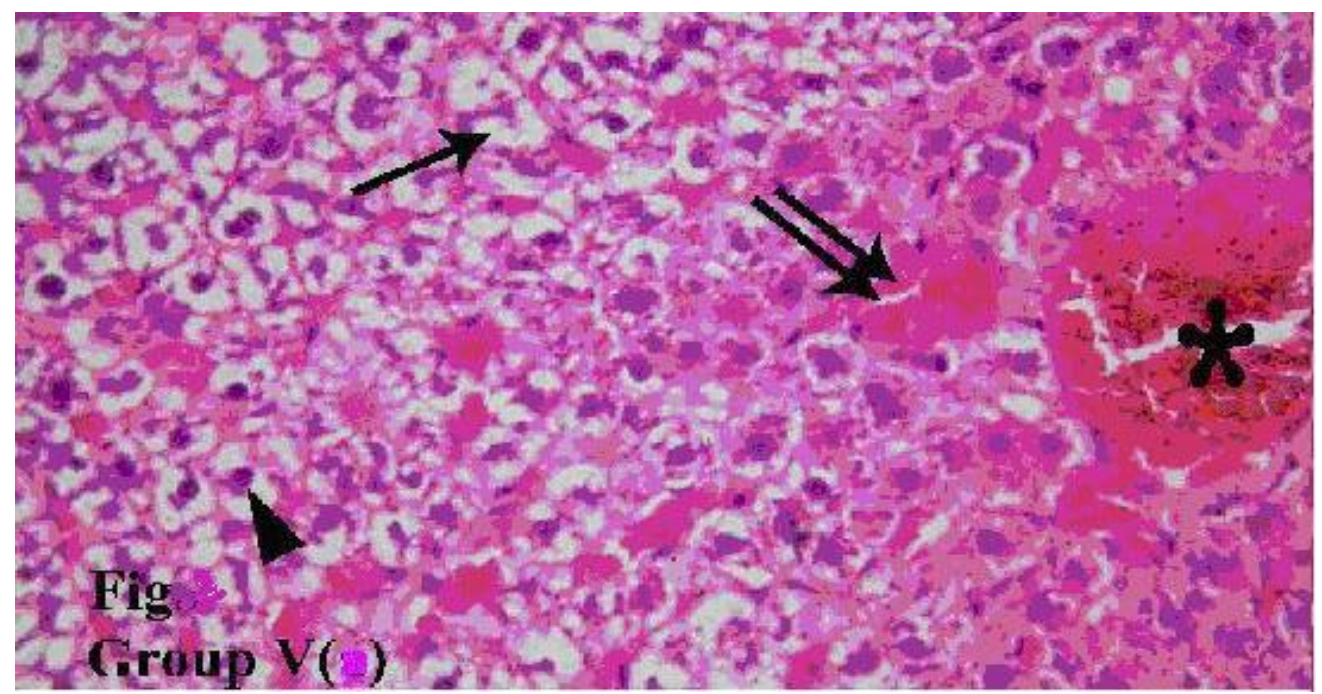

Figure 8 photomicrographs of rat liver sections (group V): showing extensive vacuolated hepatocytes ( $\uparrow$ ), with abnormal dense nuclei $(\Delta)$. Congested central vein $(*)$ and hepatic sinusoids $(\uparrow \uparrow)$ can also be $\operatorname{detected}(H \& E X$ 640) 


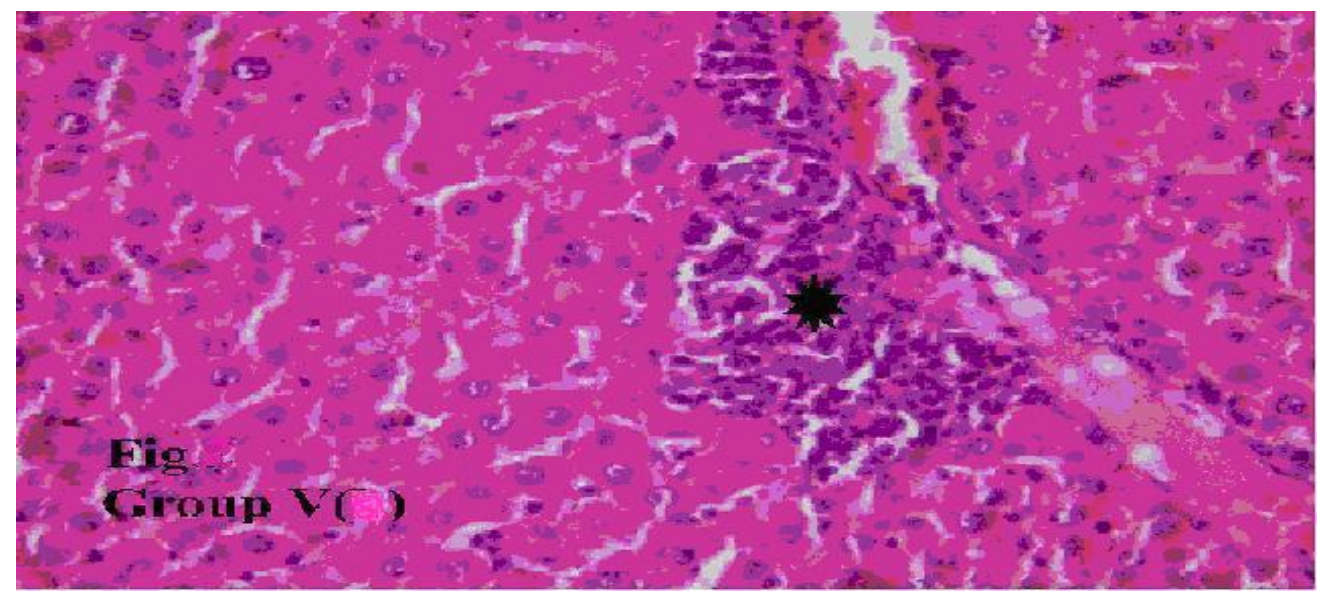

Figure 9 photomicrographs of rat liver sections (group V): showing area of mononuclear cellular infiltration (*).(H\&E X 640).

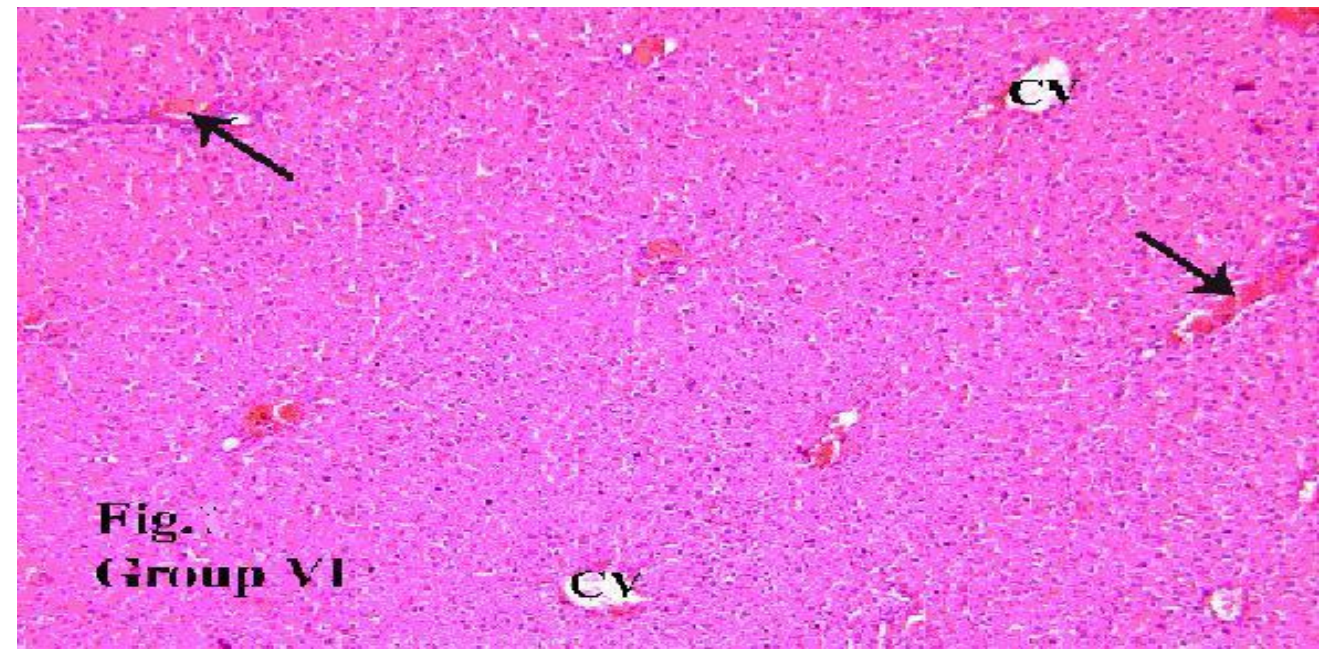

Figure 10: photomicrographs of rat liver sections. (group VI): showing central veins and congestion of the portal vein $(\uparrow)$ in the portal tract. $(H \& E X 250)$.

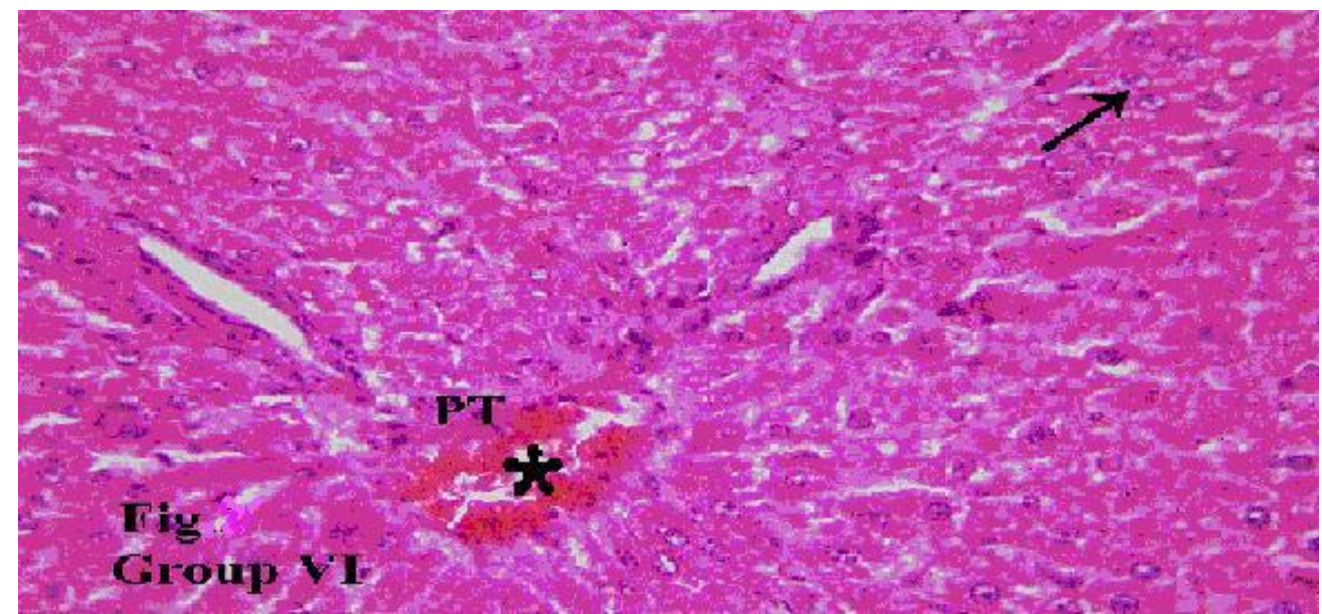

Figure 11 photomicrographs of rat liver sections (group VI): : showing mild dilatation of the portal tract (PT) with congestion of the portal vein $(*)$. Hepatocytes are seen with vesicular nuclei ( $\uparrow$ ) with no cytoplasmic vacuolations(H\&E X 640). 


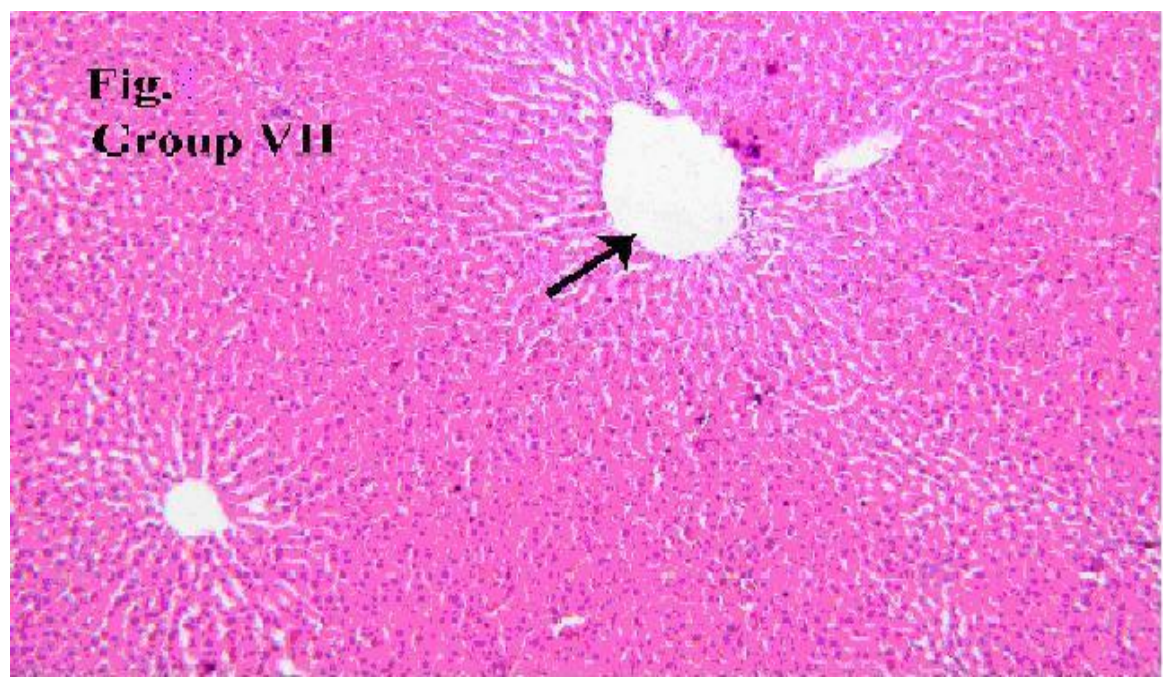

Figure 12: photomicrographs of rat liver sections (group VII): showing dilated non congested portal vein ( $\uparrow) .(H \& E X 250)$.

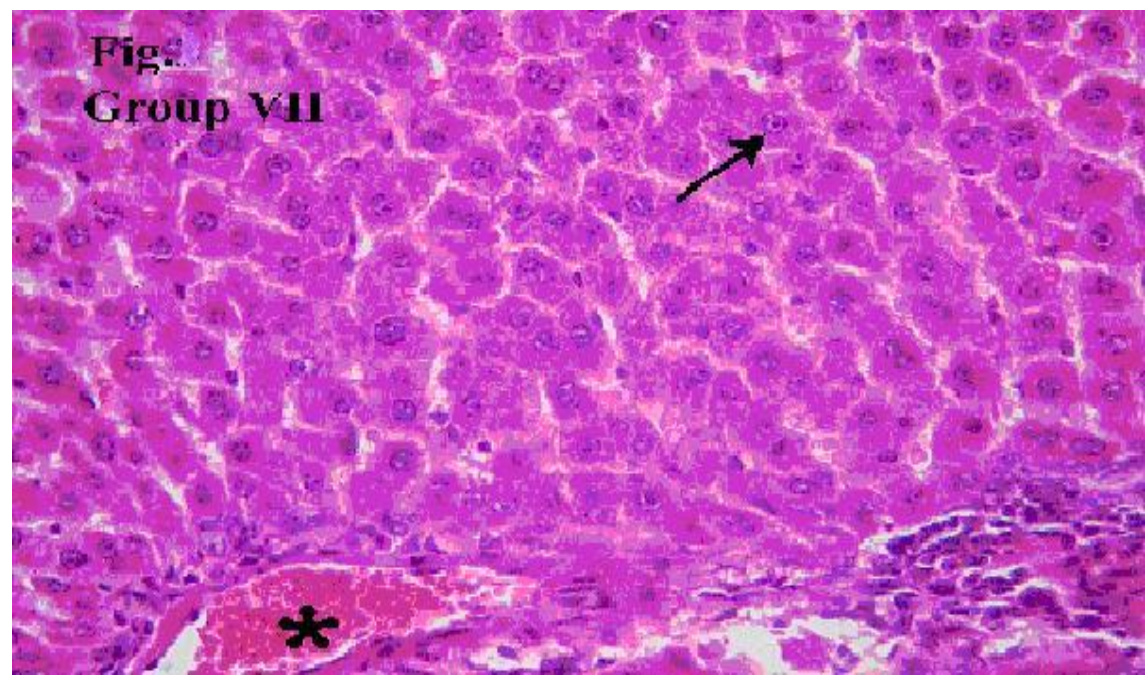

Figure 13 photomicrographs of rat liver sections (group VII): showing mild congestion of the portal vein $(*)$. Vesicular nuclei ( $\uparrow$ ) can be also be seen. (H\&E X 640)

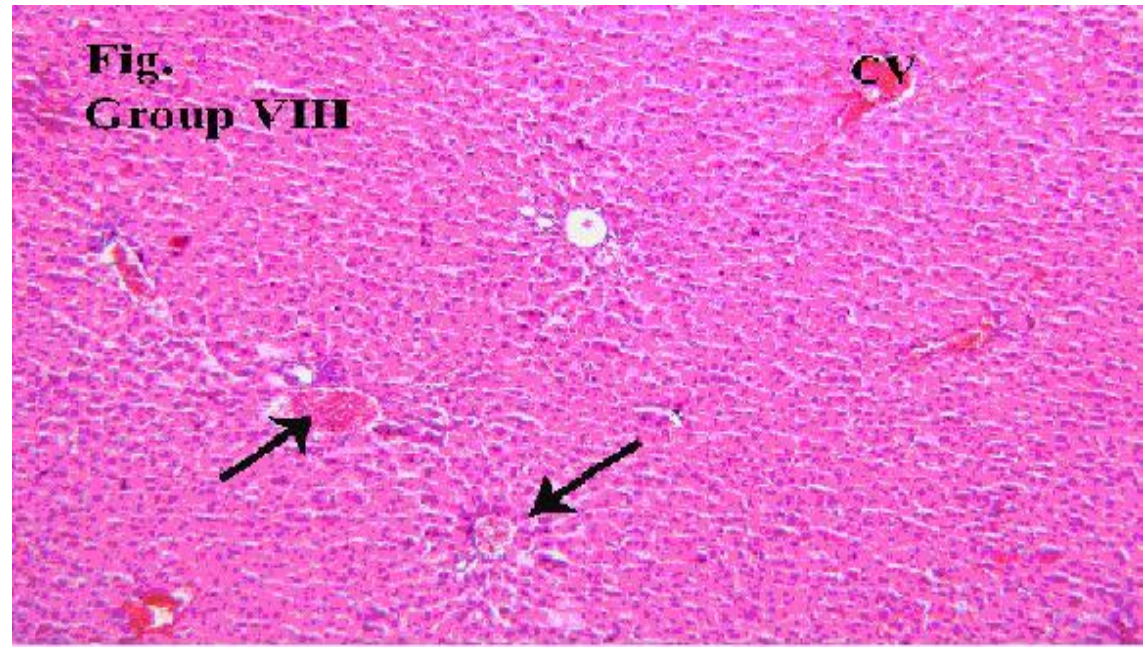

Figure 14: photomicrographs of rat liver sections (group VIII): showing congestion of the central vein (CV) and some portal tracts $(\uparrow)(H \& E X 250)$. 


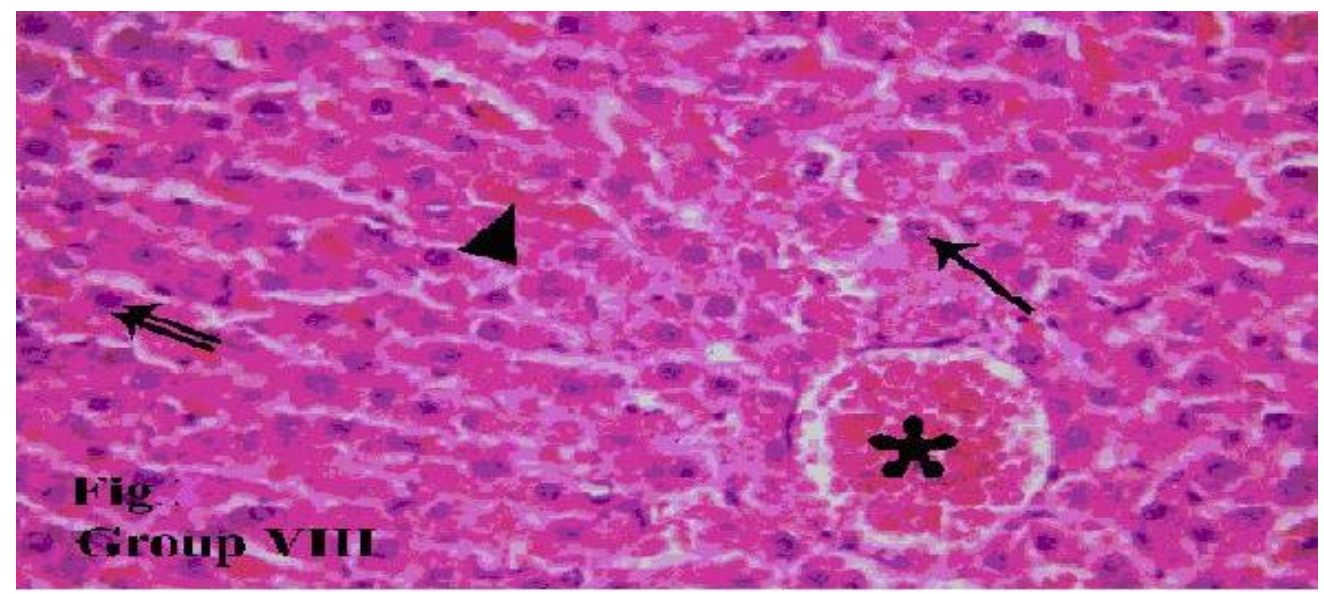

Figure 15 photomicrographs of rat liver sections. (group VIII): showing congestion in the central vein (*) and hepatic sinusoids $(\Delta)$. Most hepatocytes are seen with vesicular nuclei $(\uparrow)$, while few are seen with dense pyknotic nuclei $(\uparrow \uparrow)$. No cytoplasmic vacuolations can be detected (H\&E X 640).

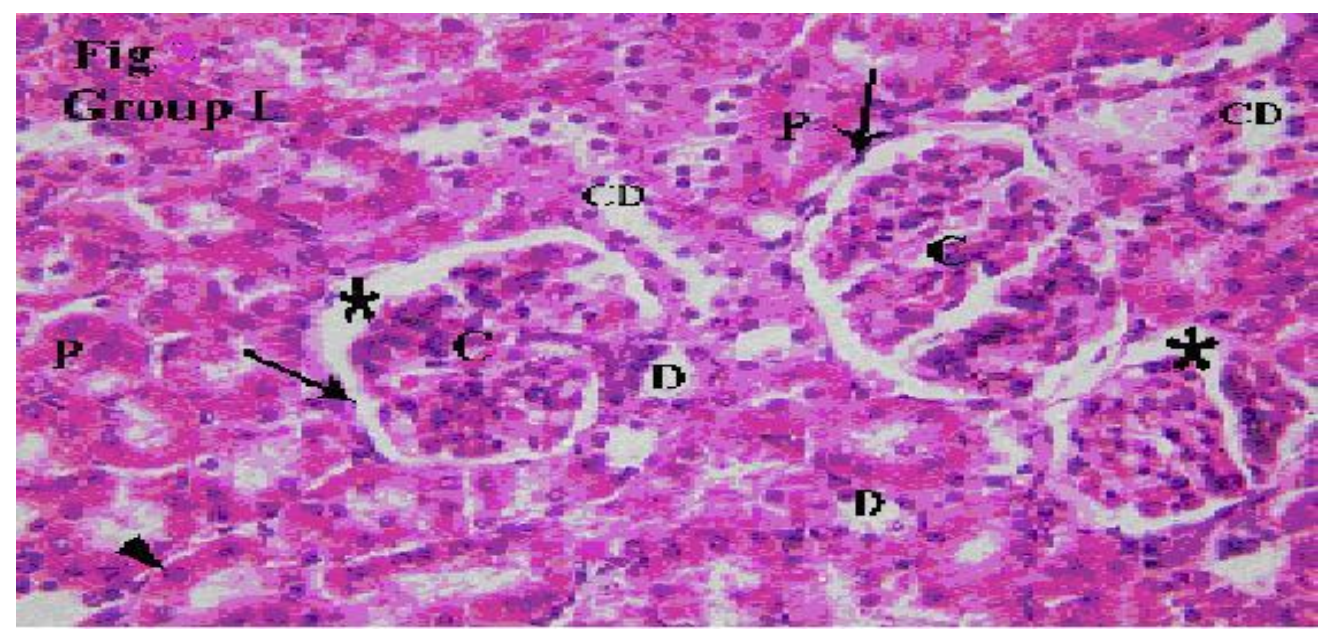

Figures 16: photomicrographs of rat kidney sections (group I): (control) showing renal corpuscles with glomerular tuft of capillaries (C) and surrounded by Bowman's capsule ( $\uparrow$ ). Filtration space $(*)$, proximal convoluted tubules (P), distal convoluted tubules (D) and cortical collecting ducts (CD) can also be seen. PCTs are seen with narrow lumina. They are lined by pyramidal cells with acidophilic cytoplasm and basal rounded vesicular nuclei. The DCTs are seen with wide lumina. They are lined with low cubical cells with pale acidophilic cytoplasm and rounded vesicular nuclei (A).(H\&E X 640).

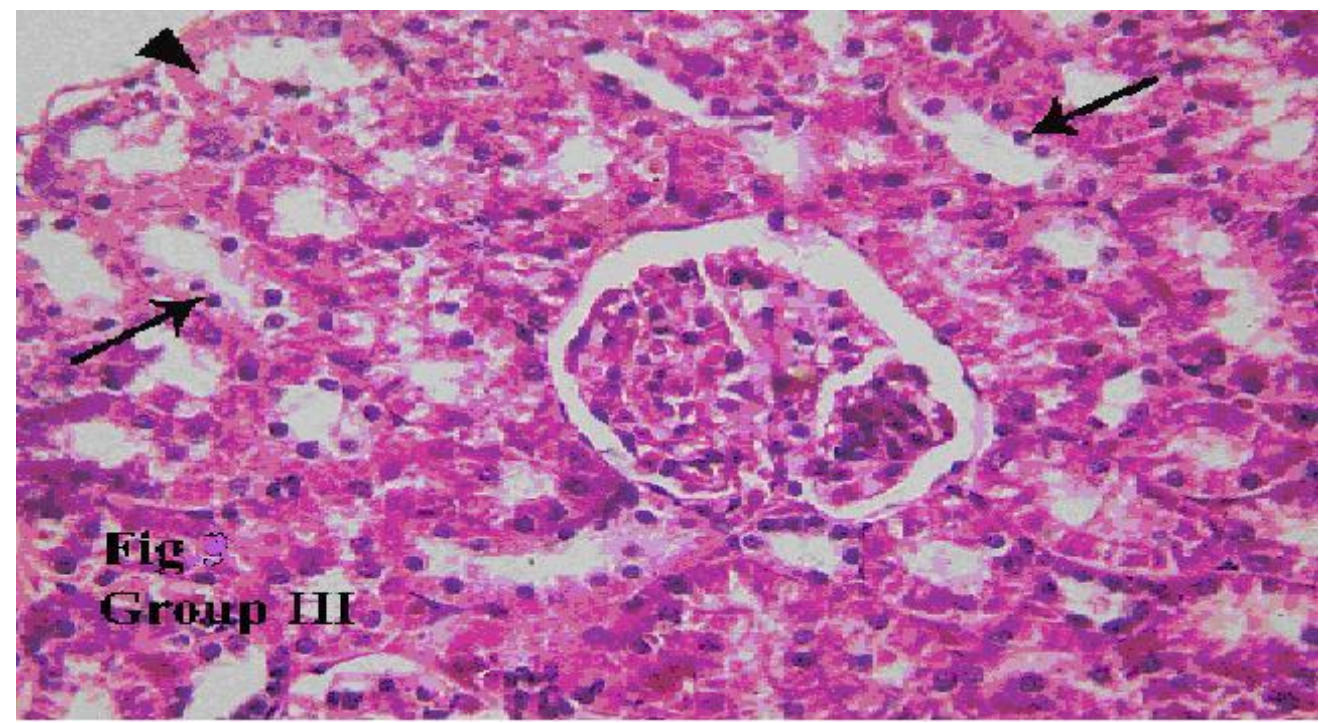

Figure 17: photomicrographs of rat kidney sections (group III): showing vacuolations of some renal tubules ( $\Delta$ ). Some tubular cells show pyknotic $(\uparrow)$ nuclei(H\&E X 640). 




Figure 18: photomicrographs of rat kidney sections (group IV): showing vacoulations of renal tubular cells ( $\Delta$ ). Pyknotic nuclei ( $\uparrow$ ) and loss of normal architecture of renal tubules can also be detected. (H\&E X 640).

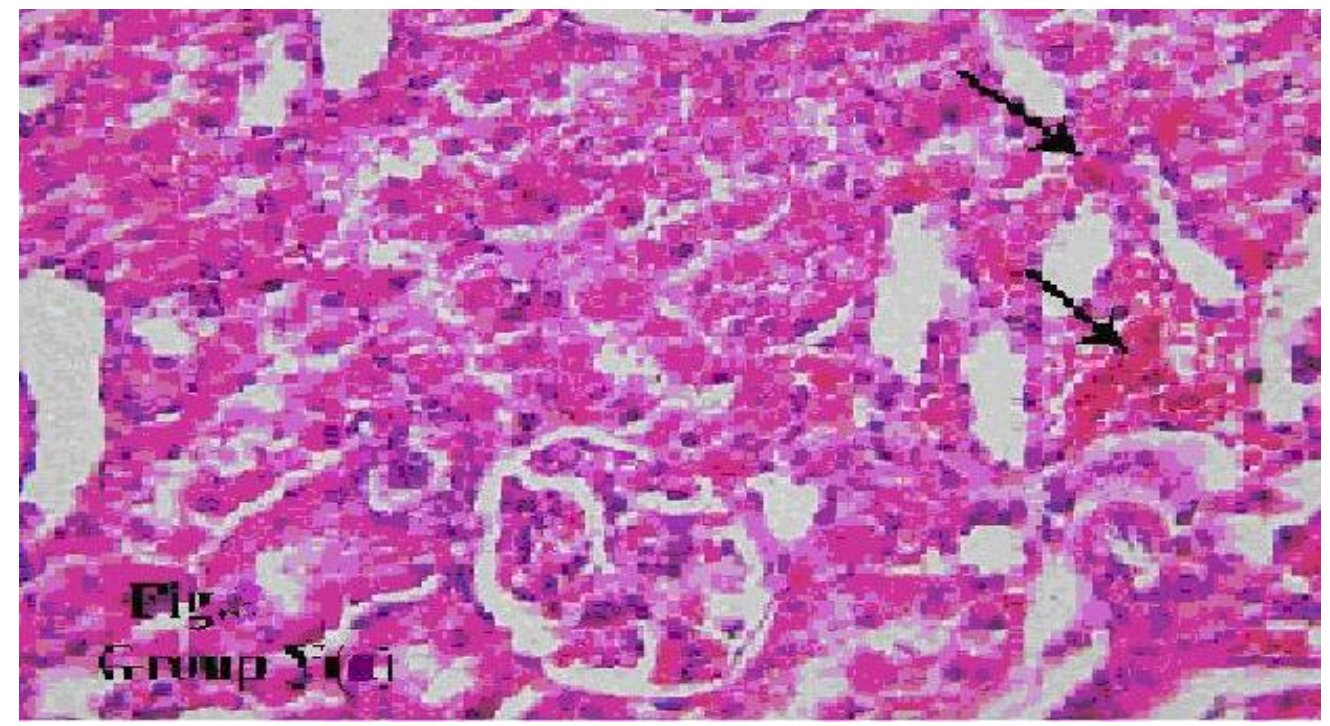

Figure 19: photomicrographs of rat kidney sections (group V): showing congestion of peritubular capillaries ( $\uparrow(H \& E X$ 640).

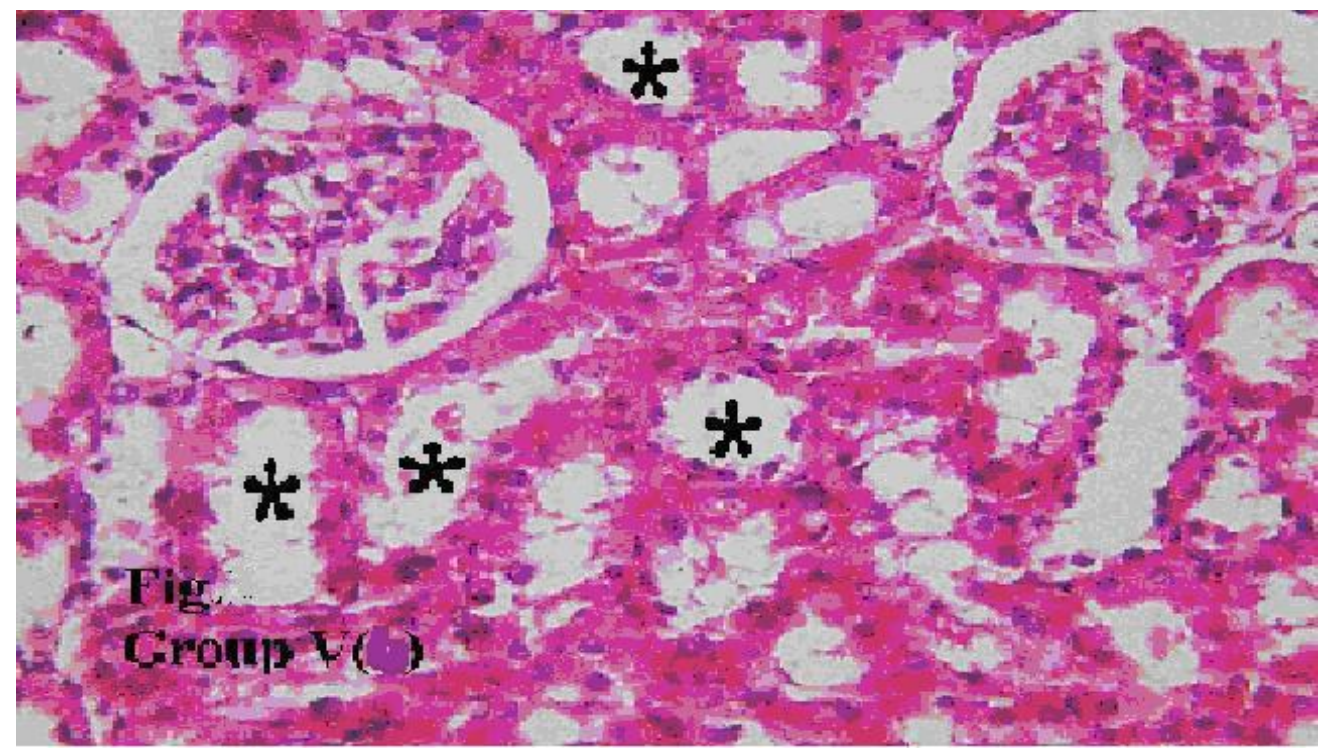

Figure 20: photomicrographs of rat kidney sections (groupV):showing loss of normal tubular architecture with dilatation of tubular lumen (*).(H\&E X 640). 


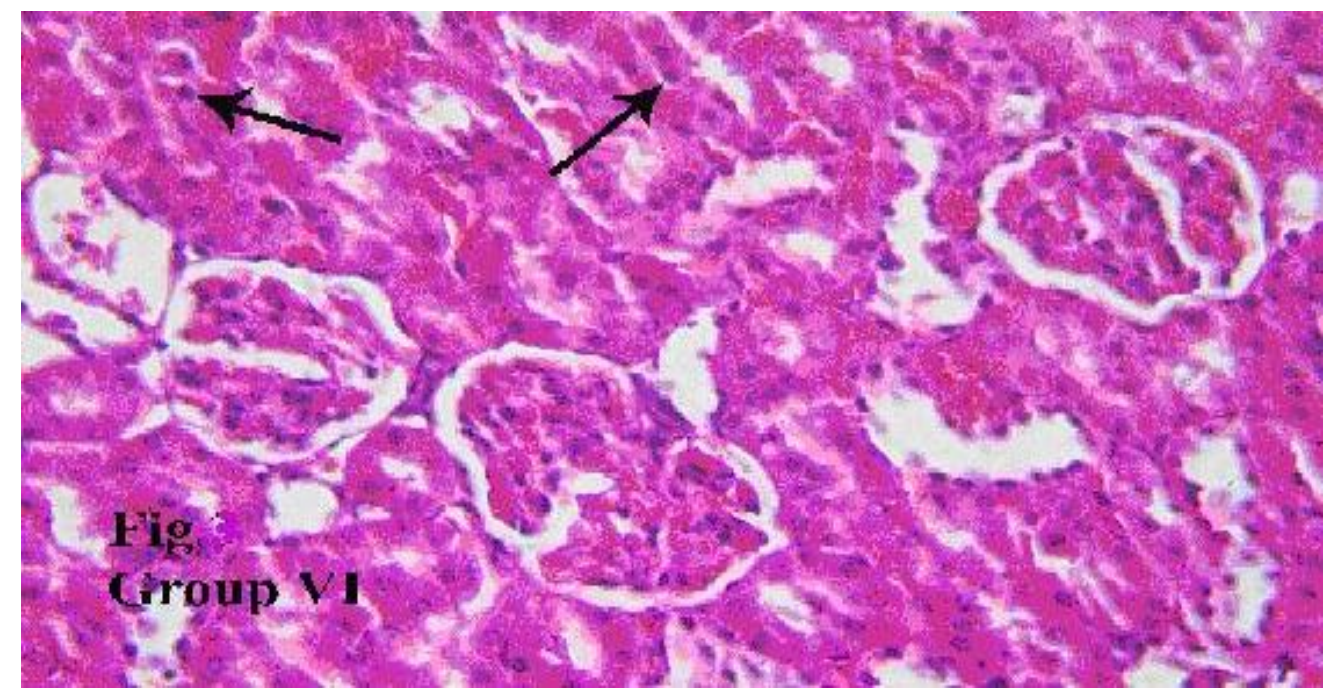

Figure 21: photomicrographs of rat kidney sections (group VI): showing few tubular cells with pyknotic nuclei (个).(H\&E X 640).

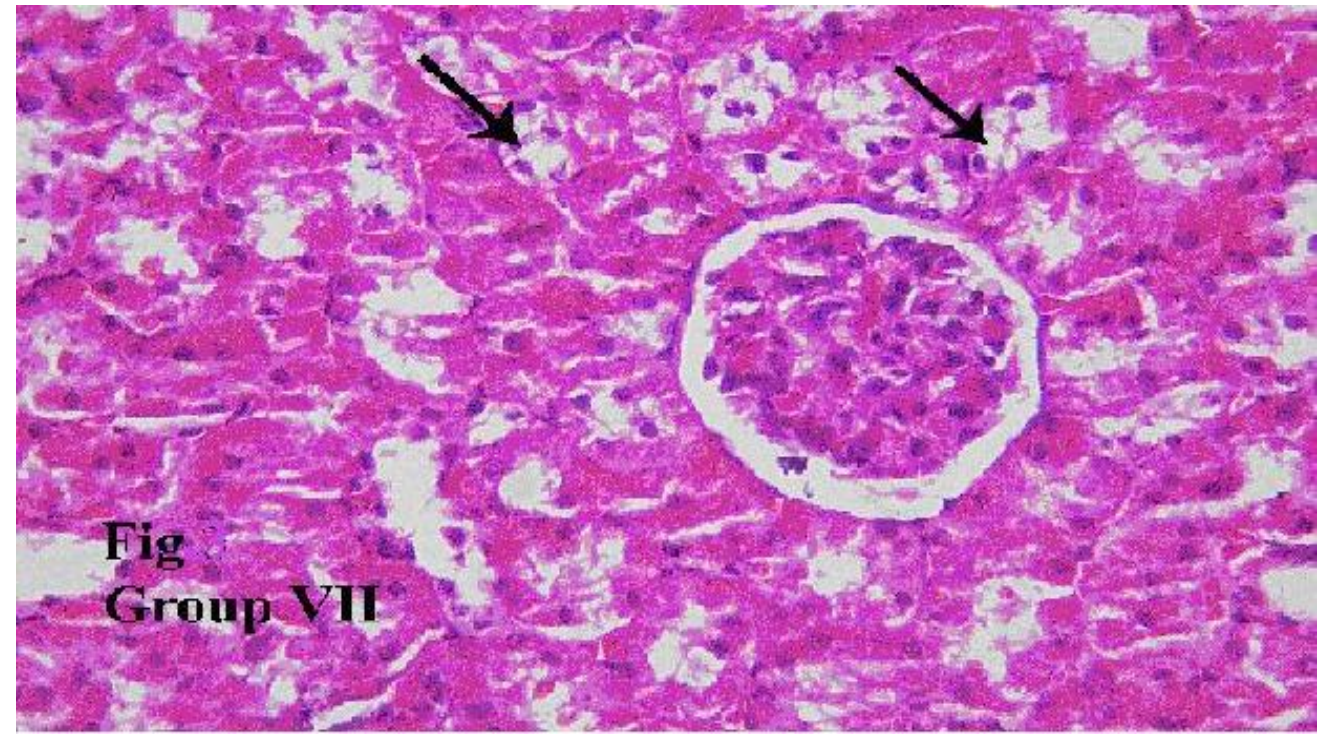

Figure 22: photomicrographs of rat kidney sections (Group VII): showing vacuolations ( $\uparrow$ ) of some renal tubular cells. (H\&E X 640).

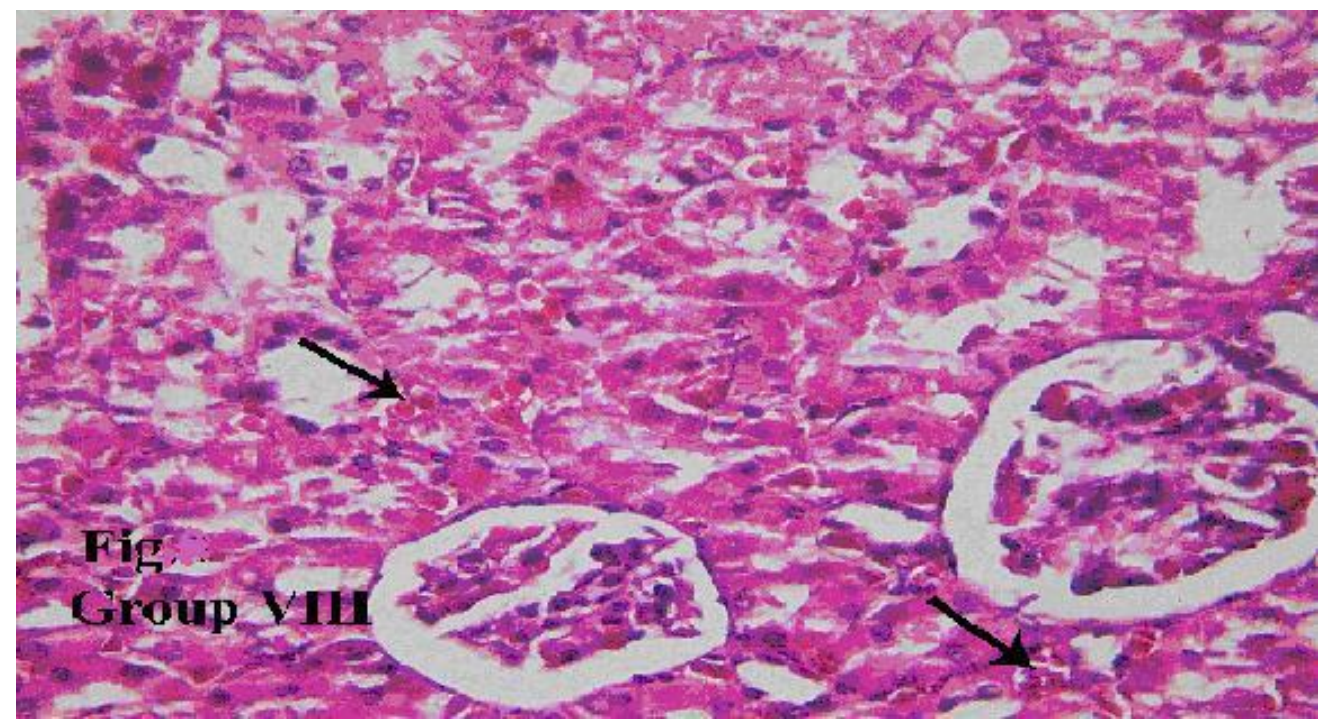

Figure 23: photomicrographs of rat kidney sections (group VIII): showing mild congestion of peritubular capillaries ( $\uparrow$ ) (H\&E X 250). 


\section{Discussion}

Chlorambucil is an alkylating antineoplastic agent indicated in treatment of lymphocytic leukemia, with serious effects including hepatotoxicity, nephrotoxicity, immune-suppression etc. (Tomenendalova et al., 2008). One of the main complications of using antineoplastic agents is the oxidative damage to normal cells during chemotherapy (Srevastava et al., 2010) This damage is due to reactive oxygen spesies which attack lipids resulting in membrane lipid peroxidation and membrane destruction (Repetto et al., 2010)

Oxidative stress and lipid peroxidation are involved in various and numerous pathological states. The term "oxidative stress" is frequently used to describe the imbalances in redox couples such as reduced to oxidized glutathione (GSH/GSSG) ratio ( Hybertson et al., 2011)

Reactive oxygen species (ROS) are thought to be the major ones responsible for the alteration of macromolecules which are often termed oxidative stress. ROS are generated as by-products of cellular metabolism, (Lu et al.,2010)

Drugs currently used in chemotherapy acting by ROS generation. Many antitumor agents exhibit antitumor activity via ROS-dependent activation of apoptotic cell death, suggesting potential use of ROS as an antitumor agent. Thus, a unique anticancer strategy named "oxidation therapy" has been developed by inducing cytotoxic oxystress for cancer treatment.(Balaram et al.,2012)

In the present study, The obtained results revealed evident hepatic damage after administration of high dose chlormabucil in the form of significant increase in ALT,AST and serum bilirubin levels as was found by Tomenendalova et al.(2008) and Summerfield et al.(2002)

The damage was proved by histopathological examination as the liver of chlorambucil treated rats for 5 days, showed vacuolated hepatocytes at the periphery of classic hepatic lobule in zone 1. Extension of the lesions was noticed in rats treated for 10 days. In rats treated for 15 days, highly vacuolated hepatocytes were noticed in zone 2 and 3 . It was reported that hepatic fat accumulation (steatosis) is characterized by small intracytoplasmic fat droplets that accumulate in the cell. It is due to a varied multitude of pathologies that disrupt normal lipid movement through the cell and cause accumulation (Hautekeete et al., 1990). Therefore un-used lipids which would normally participate in lipoprotein synthesis begin to accumulate (Conde et al., 1993) .

The present results also showed marked renal dysfunction after administration of high dose chlormabucil in the form of significant elevation of blood urea nitrogen and serum createnine as was reported by Tomenendalova et al. (2008) and Blank et al.(1983)

Histopathological examination showed vacoulation and loss of normal tubal architecture in kidney of chlormabucil treated rats. Extension of the lesion was noticed in rats treated for 10 days and 15 days. It was reported that alteration in integrin-which anchor tubal cells to basement membrane- led to detachment of the cells from the basement membrane and their shedding inside the lumen (Kumar et al., 2013), this might be the cause of loss of normal tubal structure observed in this study. This also was reported by Ramadori and Cameron (2010) and Tunde et al. (2014) who reported dose dependent histological renal damage.

To prevent the damage from ROS, cells possess several antioxidant enzymes such as antioxidant defense mechanism includes nonenzymatic antioxidants such as glutathione (GSH), which functions in the cellular thiol/disulfide system (Misra et al., 2009). It is a non enzymatic antioxidant and the primary regulatory of redox status in all cells as it is involved in scavenging hydroxyle radical and detoxification of hydrogen peroxide and lipid peroxides (Kern and Kehrer, 2005)

In the present study, there was significant reduction in hepatic and renal glutathione levels after administration of high dose chlormabucil. This was reported also by Tunde et al. (2014).

Co-administration of antioxidants with antineoplastic agents is important in reducing the toxicity of the agents (Alexieva et al., 2010), protecting normal tissues and enhancing the killing capabilities of cancer cells(Charles et al.,2007) and (Hart, 2012). Lascorbic acid is a good free radical scavenger due to its chemical properties (Dometrovic, 2006), it is one of the most important lines of antioxidant defense in hepatocytes and other cells (Schafer and Buettner, 2001) In addition, Ahmed et al .2013) and Mamede et al .(2011) observed that vitamins have been reported to play an important role in oxidative stress and cancer therapy.

In the present study, it was observed that Co treatment with L-ascorbic acid offered significant prevention of chlorambucil toxicity evidenced by significant reduction in ALT, AST and serum bilirubin levels as well as blood urea nitrogen and serum createnine levels as was found by Tunde et al. (2014) and Alexieva et al.(2010) There was also significant elevation of hepatic and renal GSH levels as was reported by Tunde et al. (2014).

This protection was evidenced also by histopathological findings, as there was significant amelioration of histopathological changes such as mild congesttion of portal tracts, few hepatocytes with pyknotic nuclei, mild congestion of peritubular capillaries, some vaculated tubular cells with pyknotic nuclei.

Although these evidenced biochemical and histopathological improvement were more obviuos with decreasing duration of treatment, there was significant protection in each group as compared with the corresponding toxic group.

\section{Conclusion}

From this study it was concluded that during treatment with high dose of chlormabucil, Co treatment with Lascorbic acid is protective against hepatic and renal toxicities induced by chlorambucil. 


\section{Recommendations}

According to this study, it is recommended to use L-ascorbic acid during treatment with chlorambucil, also, further researches are required to study the protective effects of using more than one antioxidant especially with long duration of chlorambucil therapy.

\section{References}

Ahmed M, Khan M, Muhammad N ,et al., (2013): Role of medicinal plants in oxidative stress and cancer. Scientific Reports 2(2):1-3.

Alexieva B, Markova T, Nikolova E ,et al., (2010): Free radicals aAntioxidants and cancer chemotherapy. A CTA Med Kini Univ 35(2): 57-65.

Anagnostopoulos A, Parameswaran N. Hari L, et al., (2008): Autologous or allogeneic stem cell transplantation in patients with Waldenstrom's macroglobulinemia .Journal of American Society for Blood and Marrow Transplantation doi:10.1016/04.010 12:845-854

Balaram P, Fang B, Fujimoto N,et al., (2012): Oxidative stress and lipid peroxidation products in cancer progression and therapy. International Scholarly Research Network ISRN Oncology, Volume, Article ID 137289, 21 pages doi:10.5402/2012/137289.

Bancroft JD, Cook HC, Turner DR, (1994): Manual of histological techniques and their diagnostic application. 2nd ed. USA: Churchill Livingstone: 179-189.

Blank D, Nanji A, Schreiber D, et al., (1983): Acute renal failure and seisures associated with chlormabucil overdose .J Toxicol Clin Toxicol 20: 361-365.

Charles B, Simon H, Nnicole L, Victoria S, (2007): Antioxidants and other nutrients do not iInterfere with chemotherapy or radiation therapy and can increase kill and increase survival .Alternative Therapies in Health and Medicine. Jan/Feb; 13,1; Research Library.

Conde A, González E Santolaria F, et al., (1993): liver changes in protein malnutrition. An Experimental Study in Rats. Nutr Hosp. 8(6): 358-363.

Dometrovic R, (2006):Vitamin C in disease prevention and therapy. Biochema Medica 16(2) :107125.

Frankel S and Gradwohl E, (1970): Colorimetric method for determination of serum transaminases. Am. J. Clin.Pathol 28: 26-34.

Ghosh J, Das J, Manna P, et al., (2010): Protective effect of the fruits of Terminalia Arjuna against cadmium-induced oxidant stress and hepatic cell injury via MAPK activation and mitochondria dependent pathway. Food Chem 123: 1062-1075.

Guigonis V, Dallocchio A, Baudouin A et al.,(2008): Rituximab treatment for severe steroid- or cyclosporinedependent nephrotic syndrome: a multicentric series of 22 cases Journal of Pediatr Nephrol (2008) 23:1269-1279.
Hart j, (2012): Data support antioxidant use during chemotherapy. Western Reserve University School of Medicine in Chlveland Ohio vol18 issue 2: 91-97.

Hautekeete M, Degott C and Benhamou J, (1990): Microvesicular steatosis of the Liver. Acta Clin Belg. 45(5): 311-26.

Hybertson B, Gao B, Bose S, et al., (2011): Oxidative stress in health and disease: the therapeutic potential of Nrf2 activation, Molecular Aspects of Medicine, vol. 32,: 234-246.

Kern J and Kehrer P, (2005): Free radical and apoptosis relationship with glutathione thioredoxin and the Bcl family of protiens .Front Biosci 10:1727-1738.

Kumar V, Abbass A and Aster J, (2013): Robbins Basic Pathology. Ninth edition. Elsevier, Saunders: 536-538.

Lawrence $M$ and Robert H,(1993):Methods of determination of blood urea nitrogen and serum creatinine, In: Tietz Textbook of Clinical Chemistry, $2^{\text {nd }}$ edition, WB Saunders Company,London,pp.621

Li X,Gou J,Yan X,et al., (2010): Chlorambucil for primary biliary cirrhosis. Cochrane Database of Systematic Reviews 9.

Lu J. Lin P. Yao Q. et al., (2010): Chemical and molecular mechanisms of antioxidants: Experimental approaches and model systems, Journal of Cellular and Molecular Medicine, vol. 14, no. 4: 840-860.

Mamede A, Tavares S and Abrantes A, (2011): The role of vitamins in cancer, Review. Nutrition and Cancer 634:479-494.

Mc Evoyed G, (2006): Drug information. American Scociety of Health-System Pharmacies Inc Betheseda Maryland: 957-978.

Misra M, Sarwat M, Bhakuni P,et al., ( 2009): Oxidative stress and ischemic myocardial syndromes, Medical ScienceMonitor, vol. 15, no. 10: RA209-RA219.

Pangalis G, Vassilakopoulos T, Dimopoulou M, et al., (2002): B-chronic lymphocytic leukemia: Practical Aspects. Hematol Oncol 20: 103-146.

Repetto M, Ferrarotti A, BoverisA, (2010): The involvement of transition metal ions on irondependent lipid peroxidation. Archives of Toxicology 84:255-262.

Ramadori G and Cameron S,(2010): Effects of systemic chemotherapy on the liver.Annals of Hepatology 9(2):133-143.

Schafer F and Buettner G, (2001): Redox environment of the cell as viewed through the redox state of the glutathione disulphide/glutathione couple. Free Radic Biol Med 30: 1191-1212.

Singh A, Baht T and Shrma O (2011):Clinical biochemistry of hepatotoxicity,J Clinic Toxicol S4: 1-19.

Srevastava, A Gupta, S Srevastava, S, et al., (2010): Cisplatin cobbination chemotherapy induces oxidative stress in Advance non-small cell lung cancer patients. Asian Pacific J Cancer 
Prev.11: (4)65-47.

Summerfield G, Taylor P, Mounter P, et al., (2002):

High dose chlorambucil for the treatment of chronic lymphocytic leukemia and low grade non hodgkin lymphoma.Br J Haematol 116: 782-786.

Suber R (1994): Clinical pathology methods for toxicology In: Principles and Methods of Toxicology. Hayes AW, (ed) $3^{\text {rd }}$, Raven Press, New York, 4:76-98.

Taylor J (1990): Statistical technique for data analysis. $2^{\text {nd }}$ Lewis Pub.Inc.US:25-30.
Trindade J, Maria M, Botelho F, (2011): The role of vitamins In cancer, a review. Nutrition and Cancer 634:479-494.

Tomendedelova J, Mayer J, Doubek M, et al., (2008): Toxicity of high -dose chlorambucil in wister rats. ACTA VET BRNO 77: 595-602.

Tunde E, Ayokanmi O, Kehinde, A (2014): Kolaviron and L-ascorbic acid ameliorate chlorambucil induced hepatic and renal toxicity in rat. International Journal of Toxicology and Applied Pharmalogy 4(1); (20): 23-32.

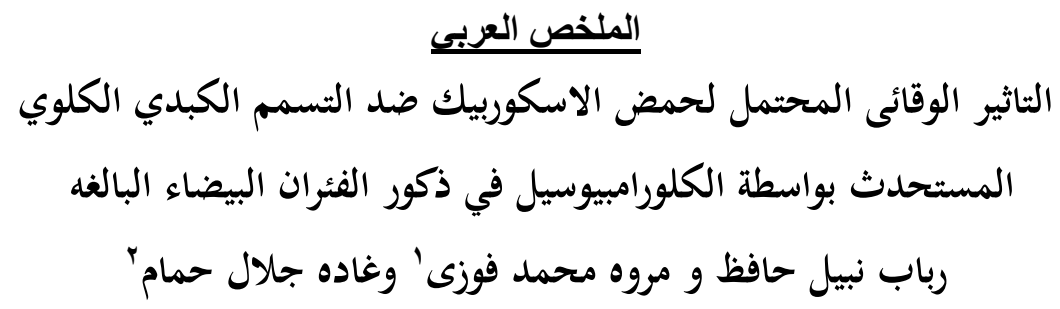

ادت النسبه العاليه للاورام السرطانيه لاستخدام العديد من الادويه المضاده للسرطان من اجل مكافحه تلك الامراض.ان العلاج بالكلورامبيوسيل بجرعه عاليه يصاحبه تسمم بعده اعضاء الغرض من هذا البحث هو تقييم الدور الوقائى المتمل لحمض الاسكوربيك الوقائى لمنع التسمم الكبدي والكلوى الناتج عن. الكلورامبيوسيل فن الفئران.

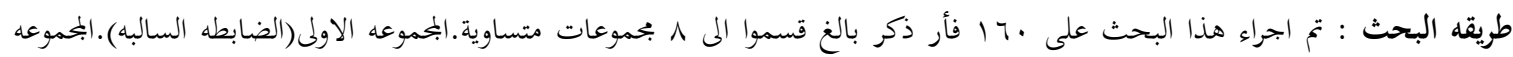

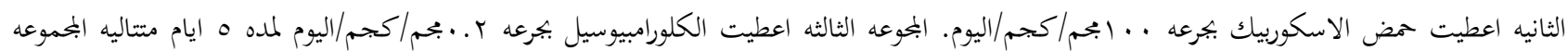

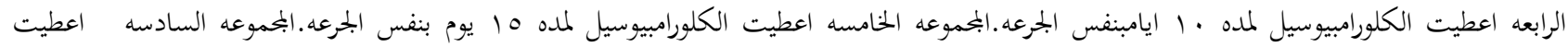

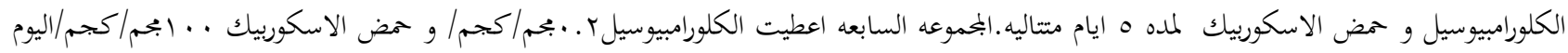
لمده • ا ايام بنفس الجرعات. البمموعه الثامنه اعطيت الكلورامبيوسيل و حض الاسكوربيك لمده 10 يوم بنفس الجرعات و تم اعطاء جميع الادوية بالفم. النتائج :بالمقارنه بالبحموعه الاولى كان هناك تغير ذو دلاله احصائيه في متوسط نتائج انزيمات الكبد و وظائف الكلى و الحكى اس اتش الكبدى بلى و الكلوي في المحموعات الثالثه والرابعه والخامسه.ايضا,كان هناك في البمموعات السادسه ,السابعه و الثامنه تغير ذو دلاله احصائيه في كل النتائج بالمقارنه بابمموعه الاولى وحين قورنت المحموعات السادسه,السابعه والثامنه مع الجموعات الثالثه,الرابعه والخامسه على التوالى اظهر ذلك تغير ذو دلاله احصائيه ايضا. اظهر فحص الانسجه للكبد تلف شديد في صوره احتقان وتوسع للاورده المركزيه و الجهاز البابي وانتفاخات بالخلايا الكبديه وانويه تغلظيه في البحموعه الثالثه هذه التغيرات موجوده بشده في المحموعتين الرابعه والخامسه والتي تميزت بوجود الخايا ذات النواه الواحده.بينما كان هناك تلف بسيط في فورون صوره احتقان بسيط

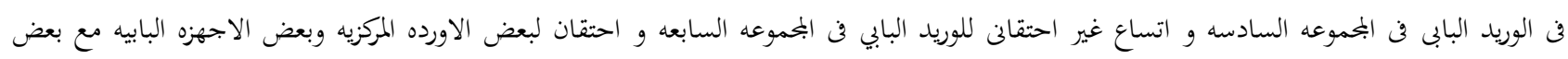
الخلايا الكبديه ذات الانويه التغلظيه في البحموعه الثامنه.علاوه على ذلك اظهر فحص الانسجه للكلى تلف شديد فئل في صوره انتفاخ لبعض الانابيب الكلويه مع

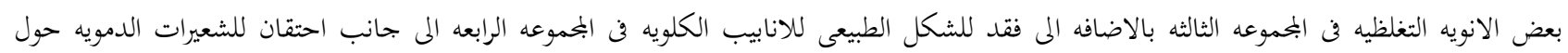
الانابيب الكلويه مع اتساع شديد بقطر الانابيب في البمموعه الخامسه.من ناحيه اخرى,كان هناك تلف بسيط في صوره عدد قليل من الخلايا الانابيبيه بانويه تغلظيه في المحموعه السادسه وانتفاخات لبعض الخلايا الانابيبه فن المجموعه السابعه و احتقان فقط للشعيرات الدمويه حول الانابيب في المجموعه الثامنه.بينما قلت نسبه التغيرات المرضيه في الكبد والكلى بنسبه احصائيه كبيره في البمموعات السادسه , السابعه والثامنه حين قورنت بالبمموعات الثالثه, الرابعه والخامسه.

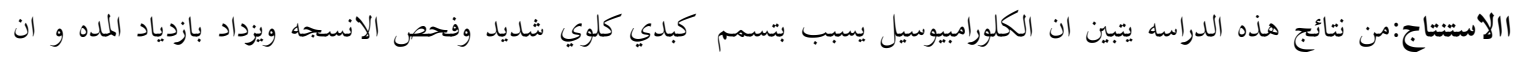
اعطاء حمض الاسكوربيك معه قد قلل من معظم تلك السميه على الكبد والكلى كما قورنت هذه الدراسه بدراسات اخرى على عدد اكبر من حيوانات

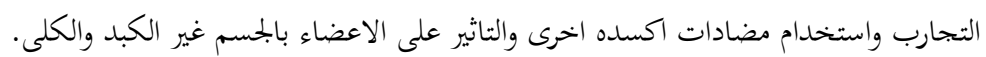

التوصيات: استخدام حمض الاسكوربيك خلال العلاج بالكلورامبيوسيل وعمل المزيد من الدراسات عن استخدام مضادات اكسده عدئ عديده معا

خاصة خلال العلاج لمده طويله بالكلورامبيوسيل .

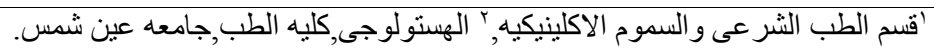

